\title{
A three-dimensional semianalytic viscoelastic model for time-dependent analyses of the earthquake cycle
}

\author{
Bridget Smith and David Sandwell \\ Institute for Geophysics and Planetary Physics, Scripps Institution of Oceanography, La Jolla, California, USA
}

Received 19 May 2004; revised 12 August 2004; accepted 31 August 2004; published 3 December 2004.

[1] Exploring the earthquake cycle for large, complex tectonic boundaries that deform over thousands of years requires the development of sophisticated and efficient models. In this paper we introduce a semianalytic three-dimensional (3-D) linear viscoelastic Maxwell model that is developed in the Fourier domain to exploit the computational advantages of the convolution theorem. A new aspect of this model is an analytic solution for the surface loading of an elastic plate overlying a viscoelastic half-space. When fully implemented, the model simulates (1) interseismic stress accumulation on the upper locked portion of faults, (2) repeated earthquakes on prescribed fault segments, and (3) the viscoelastic response of the asthenosphere beneath the plate following episodic ruptures. We verify both the analytic solution and computer code through a variety of 2-D and 3-D tests and examples. On the basis of the methodology presented here, it is now possible to explore thousands of years of the earthquake cycle along geometrically complex 3-D fault systems. INDEX TERMS: 1206 Geodesy and Gravity: Crustal movements-interplate (8155); 1242 Geodesy and Gravity: Seismic deformations (7205); 3210 Mathematical Geophysics: Modeling; 8164 Tectonophysics: Stresses — crust and lithosphere; 8120 Tectonophysics: Dynamics of lithosphere and mantlegeneral; KEYWORDS: viscoelastic deformation models, 3-D analytic deformation models, earthquake cycle

Citation: Smith, B., and D. Sandwell (2004), A three-dimensional semianalytic viscoelastic model for time-dependent analyses of the earthquake cycle, J. Geophys. Res., 109, B12401, doi:10.1029/2004JB003185.

\section{Introduction}

[2] Long-term tectonic loading, instantaneous fault rupture, and transient postseismic rebound are key components of the earthquake cycle that expose important spatial and temporal characteristics of crustal deformation. Understanding these dynamics for complicated continental transform boundaries requires three-dimensional (3-D), timedependent models that are able to simulate deformation over a wide range of spatial and temporal scales. Such ideal models must capture both the 3-D geometry of real fault systems and the viscoelastic response of repeated earthquakes. Even by limiting the problem to the quasi-static case (i.e., no seismic waves), such models must include timescales ranging from the rupture duration $(\sim 100 \mathrm{~s})$ to the vertical rebound timescale (>1000 years) and length scales ranging from the fault thickness $(\sim 500 \mathrm{~m})$ to the length of the transform boundary $(\sim 1000 \mathrm{~km})$. Purely numerical algorithms, implemented on even the most powerful computers, cannot adequately resolve these wide-range lengthand timescales. Therefore improved analytic methods are needed to reduce the scope of the numerical problem.

[3] Here we develop a semianalytic solution for the response of an elastic plate overlying a viscoelastic halfspace due to time-dependent point body forces (Figure 1). Our solution extends the analytical approach of Rundle and Jackson [1977], while enhancing computational efficiency

Copyright 2004 by the American Geophysical Union. 0148-0227/04/2004JB003185\$09.00 and maintaining qualitative agreement with many purely numerical studies. The 3-D problem is solved analytically in both the vertical dimension $(z)$ and the time dimension $(t)$, while the solution in the two horizontal dimensions $(x, y)$ is developed in the Fourier transform domain to exploit the efficiency offered by the convolution theorem. Using this numerical approach, the horizontal fault pattern and slip distribution can be arbitrarily complex without increasing the computational burden. The full 3-D time-dependent model presented here can be comfortably implemented on a desktop computer using a grid that spans spatial scales ranging from $1 \mathrm{~km}$ to $2048 \mathrm{~km}$, although larger grids are possible.

[4] In this paper we develop the semianalytic solutions to the 3-D viscoelastic problem for a vertical fault model and compare numerical results against known analytic solutions in order to assess the accuracy and efficiency of the technique. In a supplemental paper (B. Smith and D. Sandwell, manuscript in preparation, 2004) we will apply this method to simulate the earthquake cycle along the San Andreas Fault system for the past several thousand years. These models are necessary as seismic and geodetic measurements have recorded only a small portion of the earthquake cycle on major fault segments, and therefore the viscoelastic response of the asthenosphere, which will introduce long-term fault-to-fault coupling, remains poorly constrained. Furthermore, postseismic deformation mechanics pose many unanswered questions relating to the rheological parameters and time-dependent relaxation processes of the Earth. 


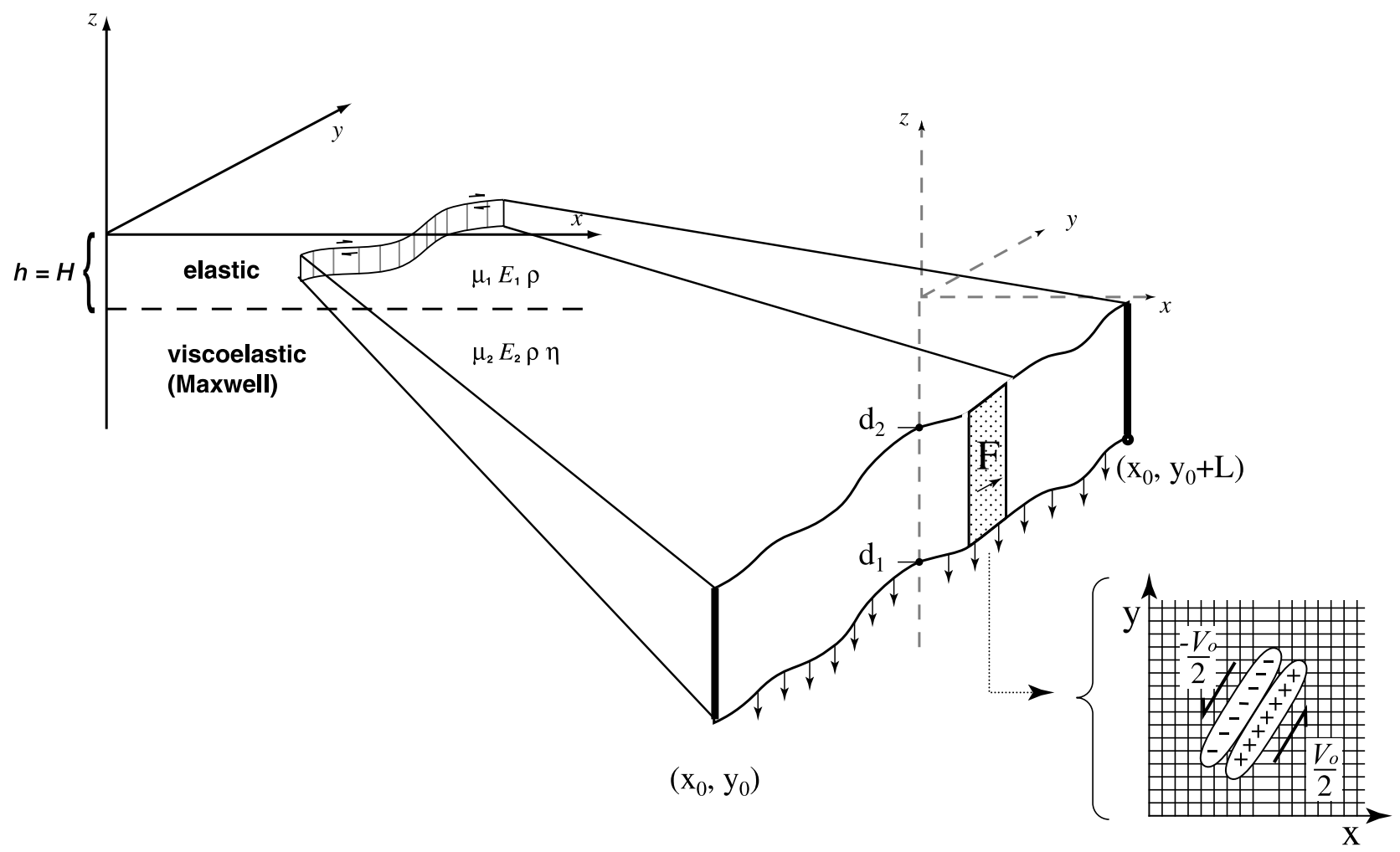

Figure 1. 3-D sketch of Fourier fault model simulating an elastic layer overlying a linear Maxwell viscoelastic half-space. Fault elements are embedded in a plate of thickness $H$ (or $h$ ) and extend from a lower depth of $d_{1}$ to an upper depth of $d_{2}$. A displacement discontinuity across each fault element is simulated using a finite width force couple, $F$, embedded in a fine grid (see Appendix E). Model parameters include plate velocity $\left(V_{o}\right)$, shear modulus $\left(\mu_{1}, \mu_{2}\right)$, Young's modulus $\left(E_{1}, E_{2}\right)$, density $(\rho)$, and viscosity $(\eta)$. Note that the elastic moduli for the viscoelastic half-space $\left(\mu_{2}, E_{2}\right)$ are time- and viscositydependent and depend upon values given to their elastic plate counterparts, $\mu_{1}$ and $E_{1}$ (Appendix D).

[5] Several postseismic models have been developed to match geodetically measured surface velocities. These models include poroelastic flow of fluids in the upper crust [e.g., Peltzer et al., 1996, 1998; Fialko, 2004a], deep afterslip [e.g., Shen et al., 1994; Heki et al., 1997; Savage and Svarc, 1997; Wdowinski et al., 1997], and fault zone collapse [e.g., Massonnet et al., 1996]. While many efforts are being made to examine these models as possible explanations of postseismic behavior, in this paper we explore a model based on viscoelastic coupling between an upper elastic plate and a lower linear viscoelastic half-space [e.g., Savage and Prescott, 1978; Thatcher, 1983, 1984; Ivins, 1996; Deng et al., 1998; Pollitz et al., 2001; Hearn et al., 2002]. We also include the restoring force of gravity in our viscoelastic model to ensure sensible vertical results. We are particularly concerned with the time-dependent vertical response of the Earth to horizontal displacements given that recent studies have shown that vertical geodetic measurements are sensitive to the thickness of the elastic later and the viscosity of the mantle [Deng et al., 1998; Pollitz et al., 2000, 2001].

[6] In addition to understanding postseismic deformation, many studies have focused on the 3-D evolution of the stress field. Coulomb stress change from large earthquakes has been used to explain the triggering of subsequent earthquakes and aftershocks [King et al., 1994; Stein et al., 1994; Kilb et al., 2000; King and Cocco, 2001; Zeng, 2001; Kilb et al., 2002; Toda et al., 2002; Anderson et al.,
2003; Fialko, 2004b]. Many stress-transfer calculations are based on purely elastic models because analytic solutions [Okada, 1985, 1992] can be computed efficiently in three dimensions for realistic fault geometries and rupture histories. However, layered viscoelastic models are needed to investigate time-dependent deformation and the triggering of earthquakes over timescales comparable to the recurrence interval. Consequently, several realistic viscoelastic models have been developed and applied to stress relaxation problems following large earthquakes [e.g., Freed and Lin, 1998; Deng et al., 1999; Kenner and Segall, 1999; Freed and Lin, 2001; Zeng, 2001; E. H. Hearn et al., manuscript in preparation, 2004]. However, because of computer speed and memory limitations, most of these numerical models are limited to a single recurrence interval and relatively simple fault geometries. Hence current models do not adequately address 3-D deformation of multiple interacting fault strands spanning multiple earthquake cycles. A complete model, incorporating both these aspects, could improve seismic hazard analyses and also provide greater insight into the physics of the earthquake cycle.

[7] While the approach we develop here is capable of addressing elaborate faulting and earthquake scenarios, it also incorporates two important improvements to the analytic model developed by Rundle and Jackson [1977]. First, we satisfy the zero-traction surface boundary condition by developing a new analytic solution to the vertical loading 
problem for an elastic plate overlying a viscoelastic halfspace, where the gravitational restoring force is included (Appendix A). The development of this analytic solution follows the approach of Burmister [1943] and Steketee [1958] but uses computer algebra to analytically invert the $6 \times 6$ matrix of boundary conditions. Second, rather than develop the Green's function for the spatial response of a point body force, we solve the differential equations and boundary conditions in the 2-D Fourier transform domain. This substantially reduces the computational burden associated with an arbitrarily complex distribution of force couples necessary for fault modeling.

[8] The remaining sections of this paper focus on the derivation of the 3-D Fourier solution, comparisons with analytic tests, and a 3-D demonstration of the earthquake cycle for a simplified fault system. In section 2 we provide a complete mathematical development of the model. This includes Appendices A-E, where many important details are provided. The computer code required to implement these equations is available at http://topex.ucsd.edu/body force. Section 3 provides a number of tests of the Fourier solution and related code against a series of analytic solutions to end-member problems (e.g., 2-D dislocations and cylindrically symmetric vertical loads). Section 4 provides several numerical examples of the time evolution of the deformation and stress fields over an earthquake cycle using simple fault geometry. These examples focus on the vertical velocity that is driven by purely horizontal dislocations as well as the temporal evolution of stress shadows following major earthquakes. Simple examples such as these provide important aspects of the earthquake cycle that will ultimately lead to a greater understanding of complex faulting scenarios spanning thousands of years.

\section{Fourier Three-Dimensional (3-D) Viscoelastic Model for Fault Deformation}

\subsection{New Developments to the Analytic Approach}

[9] At present, there are a variety of analytical and numerical 2-D and 3-D models used to investigate the behavior of elastic and viscoelastic deformation. Commonly used 2-D models include analytic solutions of Weertman [1964], Rybicki [1971], Nur and Mavko [1974], and Savage and Prescott [1978], and analytic 3-D solutions include those of Rundle and Jackson [1977] and Okada [1985, 1992]. More advanced 3-D numerical methods such as finite element models [e.g., Lysmer and Drake, 1972; Yang and Toksoz, 1981; Melosh, 1983; Cohen, 1984; Williams and Richardson, 1991], boundary element models [e.g., Crouch and Starfield, 1983; Zang and Chopra, 1991; Thomas, 1993], finite difference models [e.g., Olsen and Schuster, 1992; Frankel, 1993], matrix propagator methods [e.g., Haskell, 1953; Singh, 1970; Sato, 1971; Ward, 1984, 1985; Wang et al., 2003], etc., have been explored more recently in order to efficiently treat large-scale deformation problems with complex boundary conditions. Numerical methods such as these provide improved computational efficiency but unfortunately lack the simplicity and speed of analytic solutions.

[10] Rundle and Jackson [1977] developed a 3-D analytic viscoelastic solution (i.e., Green's function) based on the dislocation solutions of Steketee [1958], Rybicki [1971], and Nur and Mavko [1974]. While the Green's function is computationally efficient for calculating displacement or stress at a few points due to slip on a small number of faults, it is less efficient for computing deformation on large grids, especially when the fault system has hundreds or thousands of segments. Because the force balance equations are linear, the convolution theorem can be used to speed the computation as follows: take the Fourier transform of the body force couples representing fault elements, multiply by the Fourier transform of the Green's function of the model, and, finally, take the inverse Fourier transform of the product to obtain the displacement or stress field. Using this approach, the horizontal complexity of the model fault system has no effect on the speed of the computation. For example, computing vector displacement and stress on a $2048 \times 2048$ grid for a fault system consisting of 400 segments and a single locking depth requires $<40 \mathrm{~s}$ of CPU time on a desktop computer. Because multiple time steps are required to fully capture viscoelastic behavior, a very efficient algorithm is needed for computing 3 -D viscoelastic models with realistic 1000 year recurrence interval earthquake scenarios in a reasonable amount of computer time (i.e., days).

[11] In addition to enhancing the computational speed of the 3-D viscoelastic problem, we have also constructed a new solution for balancing normal stress in a layered half-space (Appendix A). The method of images is commonly used to solve continuum mechanics problems having a free surface boundary condition. An image source is used to cancel the surface shear traction, although in three dimensions the vertical traction remains nonzero. Steketee [1958] showed how to balance this vertical traction by adding a complementary solution corresponding to a vertical load on an elastic half-space: the Boussinesq problem [Boussinesq, 1885]. Rundle and Jackson [1977] used this elastic half-space solution to approximately balance the normal traction in the layered model and noted small depth-dependent errors associated with this approximation; they were chiefly concerned with horizontal deformation in their model. Burmister [1943] solved the surface loading problem for a plate overlying an elastic half-space but assumed an incompressible solid (Poisson's ratio $v=0.5$ ). While our approach is similar to that of Burmister [1943], we solve the more general layered Boussinesq problem without any restrictions on Poisson's ratio and have also included the restoring force of gravity.

[12] The full solution for the layered Boussinesq-like problem is provided in Appendix A. The important aspects of the derivation are related to the boundary conditions: (1) a vertical point load is applied at the free surface; (2) the two components of stress (normal and shear) as well as the two components of displacement (vertical and horizontal) must be continuous across the boundary between the layer and the half-space; and (3) at infinite depth, stresses and displacements within the half-space must go to zero. In the Fourier transform domain the differential equations and boundary conditions simplify to a $6 \times 6$ system of algebraic equations (A12). This system was initially inverted using computer algebra, resulting in many pages of computergenerated equations. These pages were simplified by hand to the solutions provided in equations (A14)-(A24). The simplified solutions were checked again using computer algebra. Finally, the computer code was tested against existing analytic solutions (section 3). The new Boussinesq solutions, combined with the mathematical solutions de- 
scribing displacements and stresses in a layered viscoelastic medium (section 2.2), form the full 3-D Fourier solution set.

\subsection{3-D Fourier Model Formulation}

[13] The Fourier model consists of a Fourier-transformed grid of body force couples (representing multiple fault elements) embedded in an elastic plate overlying a viscoelastic half-space (Figure 1). We begin by solving for the displacement vector $\mathbf{u}(x, y, z)$ due to a point vector body force at depth. The following text provides a brief outline of our mathematical formulation, while the full derivation and source code are available at http://topex.ucsd.edu/body_ force.

[14] 1. Develop differential equations relating a 3-D vector body force to a 3-D vector displacement:

$$
\begin{aligned}
& \mu \nabla^{2} u+(\lambda+\mu)\left[\frac{\partial^{2} u}{\partial x^{2}}+\frac{\partial^{2} v}{\partial y d x}+\frac{\partial^{2} w}{\partial z \partial x}\right]=-\rho_{x} \\
& \mu \nabla^{2} v+(\lambda+\mu)\left[\frac{\partial^{2} u}{\partial x \partial y}+\frac{\partial^{2} v}{\partial y^{2}}+\frac{\partial^{2} w}{\partial z \partial y}\right]=-\rho_{y} \\
& \mu \nabla^{2} w+(\lambda+\mu)\left[\frac{\partial^{2} u}{\partial x d z}+\frac{\partial^{2} v}{\partial y d z}+\frac{\partial^{2} w}{\partial z^{2}}\right]=-\rho_{z}
\end{aligned}
$$

where $u, v$, and $w$ are vector displacements, $\lambda$ and $\mu$ are Lame parameters, and $\rho_{x}, \rho_{y}$, and $\rho_{z}$ are vector body force components. A vector body force is applied at $x=y=0, z=$ $a$. Note that $z$ is positive upward, and $a$ has a value less than zero. To partially satisfy the boundary condition of zero shear traction at the surface, an image source [Weertman, 1964] is applied at a mirror location at $x=y=0, z=-a$ :

$$
\rho(x, y, z)=\mathbf{F} \delta(x) \delta(y) \delta(z-a)+\mathbf{F} \delta(x) \delta(y) \delta(z+a) .
$$

[15] 2. Take the 3-D Fourier transform of equations (1) and (2) to reduce the partial differential equations to a set of linear algebraic equations.

[16] 3. Invert the linear system of equations to obtain the 3-D displacement vector solution for $U(\mathbf{k}), V(\mathbf{k})$, and $W(\mathbf{k})$ :

$$
\begin{aligned}
& {\left[\begin{array}{c}
U(\mathbf{k}) \\
V(\mathbf{k}) \\
W(\mathbf{k})
\end{array}\right]=\frac{(\lambda+\mu)}{|\mathbf{k}|^{4} \mu(\lambda+2 \mu)}} \\
& {\left[\begin{array}{ccc}
\left(k_{y}^{2}+k_{z}^{2}\right)+\frac{\mu|\mathbf{k}|^{2}}{(\lambda+\mu)} & -k_{y} k_{x} & -k_{z} k_{x} \\
-k_{x} k_{y} & \left(k_{x}^{2}+k_{z}^{2}\right)+\frac{\mu|\mathbf{k}|^{2}}{(\lambda+\mu)} & -k_{z} k_{y} \\
-k_{x} k_{z} & \left(k_{x}^{2}+k_{y}^{2}\right)+\frac{\mu|\mathbf{k}|^{2}}{(\lambda+\mu)}
\end{array}\right]} \\
& \cdot \frac{\left(e^{-i 2 \pi k_{z} a}+e^{i 2 \pi k_{z} a}\right)}{4 \pi^{2}}\left[\begin{array}{c}
F_{x} \\
F_{y} \\
F_{z}
\end{array}\right],
\end{aligned}
$$

where $\lambda$ and $\mu$ are elastic constants, $\mathbf{k}=\left(k_{x}, k_{y}, k_{z}\right),|\mathbf{k}|^{2}=$ $\mathbf{k} \cdot \mathbf{k}$, and where exponents raised to the power of $\pm i 2 \pi k_{z} a$ correspond to the image and source components, respectively.
[17] 4. Perform the inverse Fourier transform in the $z$ direction (depth) by repeated application of the Cauchy residue theorem. In the following equation, $\mathbf{U}(\mathbf{k}, z)$ represents the deformation matrix, where $|\mathbf{k}|=\left(k_{x}^{2}+k_{y}^{2}\right)^{1 / 2}$ and subscripts $s$ and $i$ refer to source and image components:

$$
\left[\begin{array}{c}
U(\mathbf{k}) \\
V(\mathbf{k}) \\
W(\mathbf{k})
\end{array}\right]=\mathbf{U}(\mathbf{k}, z)=\mathbf{U}_{s}(\mathbf{k}, z-a)\left[\begin{array}{c}
F_{x} \\
F_{y} \\
F_{z}
\end{array}\right]+\mathbf{U}_{i}(\mathbf{k},-z-a)\left[\begin{array}{c}
F_{x} \\
F_{y} \\
F_{z}
\end{array}\right]
$$

[18] 5. Introduce a layer of thickness $H$ into the system through an infinite summation of image sources [Weertman, 1964; Rybicki, 1971], reflected both above and below the surface $z=0$ (Appendix B):

$$
\begin{gathered}
{\left[\begin{array}{c}
U(\mathbf{k}) \\
V(\mathbf{k}) \\
W(\mathbf{k})
\end{array}\right]=\mathbf{U}_{s}(\mathbf{k}, z-a)\left[\begin{array}{c}
F_{x} \\
F_{y} \\
F_{z}
\end{array}\right]+\mathbf{U}_{i}(\mathbf{k},-z-a)\left[\begin{array}{c}
F_{x} \\
F_{y} \\
F_{z}
\end{array}\right]} \\
+\sum_{m=1}^{\infty}\left(\frac{\mu_{1}-\mu_{2}}{\mu_{1}+\mu_{2}}\right)^{m}\left[\begin{array}{c}
\mathbf{U}_{i}(\mathbf{k}, z-a-2 m H) \\
+\mathbf{U}_{i}(\mathbf{k},-z-a+2 m H) \\
+\mathbf{U}_{i}(\mathbf{k}, z-a+2 m H) \\
+\mathbf{U}_{i}(\mathbf{k},-z-a-2 m H)
\end{array}\right]\left[\begin{array}{c}
F_{x} \\
F_{y} \\
F_{z}
\end{array}\right] .
\end{gathered}
$$

In equation (5), shear moduli $\mu_{1}$ and $\mu_{2}$ refer to the elastic constants of the layer and underlying half-space, respectively. The development of this solution requires an infinite number of image sources, $m$, to satisfy the stress-free surface and layer boundary conditions, and therefore convergence of the series in the case $\mu_{2}=0$ is problematic. This special case, which corresponds to the end-member case of an elastic plate overlying a fluid half-space, is solved in Appendix C.

[19] 6. Integrate the point source Green's function over depths $\left[d_{1}, d_{2}\right]$ to simulate a fault plane (equation (6)). For the general case of a dipping fault, this integration can be done numerically. However, if the fault is vertical, the integration can be performed analytically. The displacement or stress can be evaluated at any depth $z>d_{1}$. In the following equation, $\mathbf{U}^{\prime}(\mathbf{k}, z)$ represents the depth-integrated solution:

$$
\begin{aligned}
& \int_{d_{1}}^{d_{2}} \mathbf{U}(\mathbf{k}, z) d z=\mathbf{U}^{\prime}(\mathbf{k}, z)=\left[\mathbf{U}_{s}^{\prime}\left(\mathbf{k}, z-d_{2}\right)-\mathbf{U}_{s}^{\prime}\left(\mathbf{k}, z-d_{1}\right)\right]\left[\begin{array}{c}
F_{x} \\
F_{y} \\
F_{z}
\end{array}\right] \\
& +\left[\mathbf{U}_{i}^{\prime}\left(\mathbf{k},-z-d_{2}\right)-\mathbf{U}_{i}^{\prime}\left(\mathbf{k},-z-d_{1}\right)\right]\left[\begin{array}{c}
F_{x} \\
F_{y} \\
F_{z}
\end{array}\right]+\sum_{m=1}^{\infty}\left(\frac{\mu_{1}-\mu_{2}}{\mu_{1}+\mu_{2}}\right)^{m} \\
& {\left[\begin{array}{c}
\left(\mathbf{U}_{i}^{\prime}\left(\mathbf{k}, z-d_{2}-2 m H\right)-\mathbf{U}_{i}^{\prime}\left(\mathbf{k}, z-d_{1}-2 m H\right)\right) \\
-\left(\mathbf{U}_{i}^{\prime}\left(\mathbf{k},-z-d_{2}+2 m H\right)-\mathbf{U}_{i}^{\prime}\left(\mathbf{k},-z-d_{1}+2 m H\right)\right) \\
+\left(\mathbf{U}_{i}^{\prime}\left(\mathbf{k}, z-d_{2}+2 m H\right)-\mathbf{U}_{i}^{\prime}\left(\mathbf{k}, z-d_{1}+2 m H\right)\right) \\
-\left(\mathbf{U}_{i}^{\prime}\left(\mathbf{k},-z-d_{2}-2 m H\right)-\mathbf{U}_{i}^{\prime}\left(\mathbf{k},-z-d_{1}-2 m H\right)\right)
\end{array}\right]} \\
& {\left[\begin{array}{c}
F_{x} \\
F_{y} \\
F_{z}
\end{array}\right]}
\end{aligned}
$$


[20] The individual elements of the source and image matrices are

$\mathbf{U}_{s}^{\prime}(\mathbf{k}, Z)=\left[\begin{array}{ccc}U_{x} & U_{y} & U_{z} \\ U_{y} & V_{y} & V_{z} \\ U_{z} & V_{z} & W_{z}\end{array}\right] \quad \mathbf{U}_{i}^{\prime}(\mathbf{k}, Z)=\left[\begin{array}{ccc}U_{x} & U_{y} & U_{z} \\ U_{y} & V_{y} & V_{z} \\ -U_{z} & -V_{z} & -W_{z}\end{array}\right]$,

where $Z$ represents all $z$-dependent terms, including all combinations of $z, d_{n}$, and $2 m H$. The six independent functions of the deformation matrix are

$$
\left[\begin{array}{c}
U_{x} \\
U_{y} \\
U_{z} \\
V_{y} \\
V_{z} \\
W_{z}
\end{array}\right]=\frac{C}{\beta^{2}}\left[\begin{array}{cc}
D+\frac{k_{y}^{2}}{|\mathbf{k}|^{2}}-\frac{k_{x}^{2}}{|\mathbf{k}|^{2}} & -\frac{k_{x}^{2}}{|\mathbf{k}|^{2}} \\
-2 \frac{k_{x} k_{y}}{|\mathbf{k}|^{2}} & -\frac{k_{x} k_{y}}{|\mathbf{k}|^{2}} \\
-i \frac{k_{x}}{|\mathbf{k}|} & -i \frac{k_{x}}{|\mathbf{k}|} \\
D+\frac{k_{x}^{2}}{|\mathbf{k}|^{2}}-\frac{k_{y}^{2}}{|\mathbf{k}|^{2}} & -\frac{k_{y}^{2}}{|\mathbf{k}|^{2}} \\
-i \frac{k_{y}}{|\mathbf{k}|} & -i \frac{k_{y}}{|\mathbf{k}|} \\
D+1 & 1
\end{array}\right]\left[\begin{array}{c}
e^{-\beta Z} \\
\beta Z e^{-\beta Z}
\end{array}\right],
$$

where

$$
D=\frac{\lambda_{1}+3 \mu_{1}}{\lambda_{1}+\mu_{1}}, \quad|\mathbf{k}|=\left(k_{x}^{2}+k_{y}^{2}\right)^{1 / 2}, \quad \beta=2 \pi|\mathbf{k}| .
$$

The solutions of equation (8) are identical to those of Smith and Sandwell [2003] but have been simplified for further manipulation of the exponential terms.

[21] 7. Analytically solve for Maxwell viscoelastic time dependence using the Correspondence Principle and assuming a Maxwell time defined by $\tau_{m}=2 \eta / \mu$ (Appendix D). Following an approach similar to that of Savage and Prescott [1978], we map time and viscosity into an implied half-space shear modulus, $\mu_{2}$. We require the bulk modulus to remain constant and thus also solve for an implied $E_{2}$.

[22] 8. Calculate the nonzero normal traction at the surface, and cancel this traction by applying an equal but opposite vertical load on an elastic layer overlying a viscoelastic half-space (Appendix A).

[23] The numerical aspects of this approach involve generating grids of vector force couples (i.e., $F_{x}, F_{y}$, and $F_{z}$ ) that simulate complex fault geometry (Appendix E), taking the 2-D horizontal Fourier transform of the grids, multiplying by the appropriate transfer functions and timedependent relaxation coefficient, and, finally, inverse Fourier transforming to obtain the desired results. Arbitrarily complex curved and discontinuous faults can easily be converted to a grid of force vectors (Figure 1). The model parameters are plate thickness $(H)$, locking depths $\left(d_{1}, d_{2}\right)$, shear modulus $(\mu)$, Young's modulus $(E)$, density $(\rho)$, gravitational acceleration $(g)$, and half-space viscosity $(\eta)$. As previously mentioned, the solution satisfies the zerotraction surface boundary condition and maintains stress and displacement continuity across the base of the plate (Appendix A). The $x$ boundary condition of constant farfield velocity difference across the plate boundary is simulated using a cosine transform in the $x$ direction. The $y$ boundary condition of uniform velocity in the far field is simulated by arranging the fault trace to be cyclic in the $y$ dimension. This fault model will be used to efficiently explore the 3-D viscoelastic response of the Earth throughout the earthquake cycle.

\section{Analytic and Numeric Tests of the Layered Viscoelastic Solution}

\subsection{2-D Analytic Comparisons}

[24] Although the solutions described in section 2.2 have been checked using computer algebra, it is necessary to verify the accuracy of our computer code through comparisons with known analytic solutions. These include 2-D analytic examples of dislocations in (1) a homogeneous elastic half-space, (2) a layered elastic half-space, and (3) a layered viscoelastic half-space. For these tests, fault slip is simulated by embedding a straight, vertical fault in the $y$ dimension of a $1 \mathrm{~km}$ spaced grid of nominal dimension $2048 \times 2048$, performing a 2-D horizontal Fourier transform of the grid, multiplying by appropriate transfer functions (equations (6)-(8)), and inverse transforming to arrive at the final solution. In the subsequent models the following parameters are used, unless otherwise specified: $V_{o}=$ $40 \mathrm{~mm} \mathrm{yr}{ }^{-1}, H=50 \mathrm{~km}, d_{2}=-25 \mathrm{~km}, \mu_{1}=28 \mathrm{GPa}$, $E_{1}=70 \mathrm{GPa}$, and $\eta=10^{19} \mathrm{~Pa} \mathrm{~s}$.

\subsubsection{Homogeneous Elastic Half-Space}

[25] First, we test the surface displacement due to an infinitely long 2-D fault in a homogeneous elastic medium that is locked between depths of $d_{1}$ and $d_{2}$ (Figure 2, solid line). The analytic solution [Weertman, 1964] is given by

$$
V(x)=\frac{V_{o}}{\pi}\left[\tan ^{-1}\left(\frac{d_{2}}{x}\right)-\tan ^{-1}\left(\frac{d_{1}}{x}\right)\right],
$$

where $V_{o}$ is fault slip rate, $d_{1}$ is lower locking depth, $d_{2}$ is upper locking depth, and $x$ is the perpendicular distance across the fault plane. When $d_{1}$ is set to minus infinity, this solution is used to describe interseismic deformation (deep slip). Comparing this solution with our Fourier model (uniform elastic properties $E_{2}=E_{1}, \mu_{2}=\mu_{1}$ ) results in an error of $0.2 \%$ (gray inset, solid line). Because the fault length is assumed to be infinite, the $x$ length of the grid must be extended (e.g., 4096 elements) to achieve even higher accuracy.

[26] In addition to this 2-D example, we have also compared the 3-D results of this model to the 3-D solutions of Okada [1985, 1992] for a finite-length dislocation in a homogeneous elastic half-space. Although not presented here, the two models are in excellent agreement for both horizontal and vertical displacements (http://topex.ucsd.edu/ body_force).

\subsubsection{Layered Elastic Half-Space}

[27] As a second test, we compare the Fourier model to the 2-D analytic solution for a dislocation in an elastic layer of shear modulus $\mu_{1}$ overlying a half-space of shear modulus $\mu_{2}$ (Figure 2, dashed line). The analytic solution for the 
2-D Analytic Model Comparisons

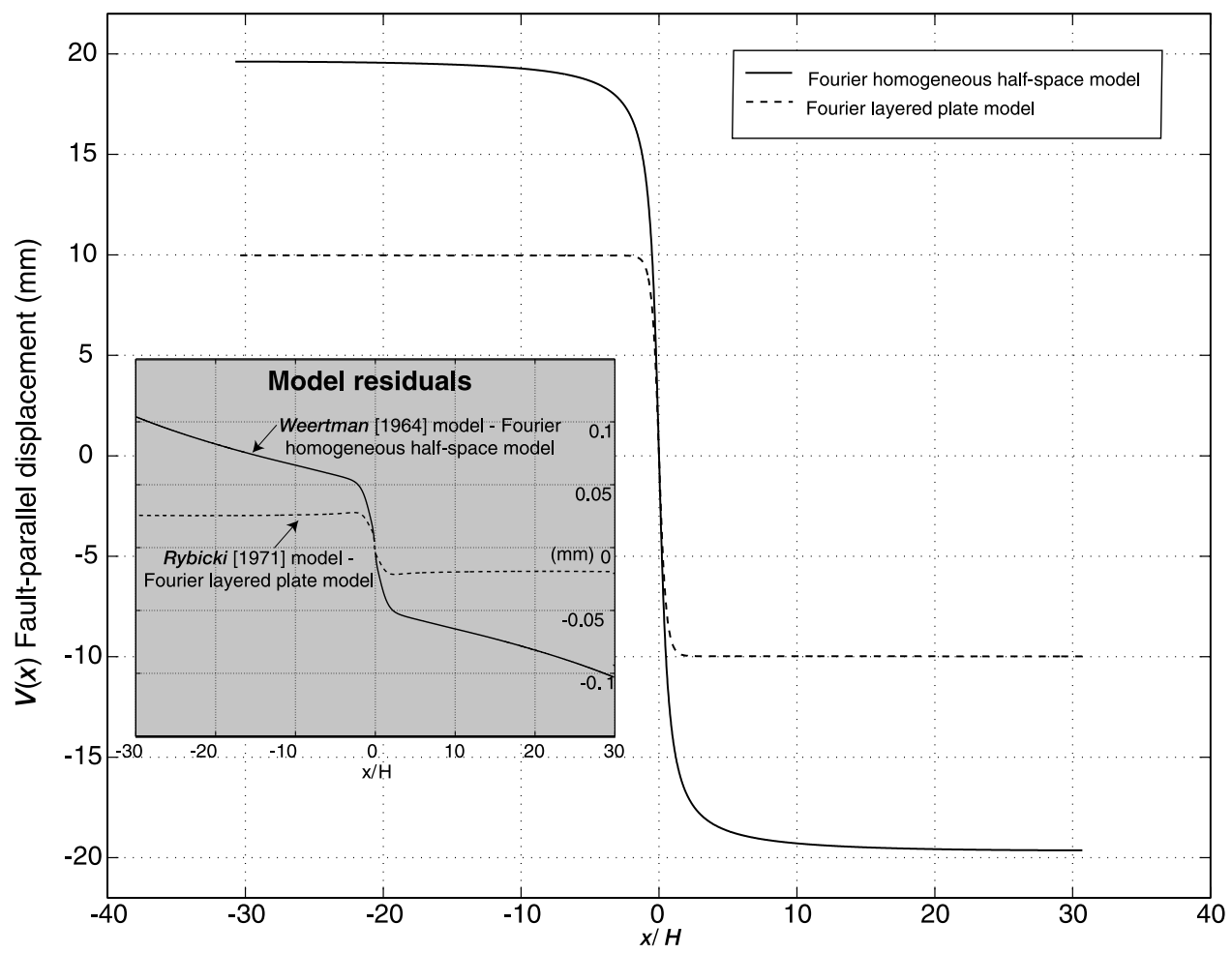

Figure 2. Comparison of fault-parallel displacement as a function of distance from the fault, $x$, with respect to plate thickness, $H$, for Fourier model profiles and existing 2-D analytic solutions. Deep fault displacement for a homogeneous half-space Fourier model is represented by the solid black line; displacement for a layered half-space model simulating an elastic plate overlying a fluid half-space is represented by the dashed black line. Note how the layered half-space model has only half the amplitude of the homogeneous half-space model due to the inherent relationship that exists between the far-field displacement and the fraction of the plate that is cracked. Both homogenous and layered half-space Fourier models have relative errors (gray inset) less than $0.2 \%$ when compared to their respective analytic solutions, the Weertman [1964] and Rybicki [1971] models, respectively. Although the Rybicki [1971] solution is limited by the number of terms $(m)$ included in the infinite series, the homogeneous half-space model comparison yields larger relative errors in the longer wavelengths, requiring a larger grid size to lower the relative error.

surface displacement due to a fault that is locked between depths of $d_{1}$ and $d_{2}$ [Rybicki, 1971] is given by

$$
\begin{aligned}
V= & \frac{V_{o}}{\pi}\left\{\tan ^{-1}\left(\frac{d_{2}}{x}\right)-\tan ^{-1}\left(\frac{d_{1}}{x}\right)+\sum_{m=1}^{\infty}\left(\frac{\mu_{1}-\mu_{2}}{\mu_{1}+\mu_{2}}\right)^{m}\right. \\
& \cdot\left[\begin{array}{l}
\tan ^{-1}\left(\frac{d_{2}-2 m H}{x}\right)-\tan ^{-1}\left(\frac{d_{1}-2 m H}{x}\right) \\
\left.\left.+\tan ^{-1}\left(\frac{d_{2}+2 m H}{x}\right)-\tan ^{-1}\left(\frac{d_{1}+2 m H}{x}\right)\right]\right\},
\end{array}\right.
\end{aligned}
$$

where $H$ is the layer thickness and $m$ refers to the number of image sources. Figure 2 (dashed line) shows the endmember case of deep slip $\left(d_{1}=-H\right)$ of a fluid half-space $\left(\mu_{2}=0\right)$. The models agree to $0.1 \%$ (gray inset, dashed line), although larger far-field deviations are possible due to the slow convergence of the Rybicki [1971] solution. We must sum more than $10^{5}$ terms of equation (10) to achieve full far-field convergence; the Fourier solution does not suffer from this convergence problem because the infinite sum is performed analytically (Appendix C).

\subsubsection{Layered Viscoelastic Half-Space}

[28] The final 2-D comparison presented here tests our implementation of the Correspondence Principle for mapping the viscoelastic properties of the model into an equivalent elastic model. In $3-\mathrm{D}$, one must be careful to maintain a time-invariant bulk modulus. The analytic solution for this model is described by Nur and Mavko [1974], although their paper does not provide the equations for mapping the Maxwell-normalized time into the rigidity of each of the image layers. This mapping is provided by Savage and Prescott [1978], although our approach differs in that we do not explicitly include a constant earthquake recurrence interval (Appendix D). We prefer to allow a variable recurrence interval to better simulate known earthquake sequences. Therefore we have no method of testing the numerical accuracy of this time-dependent model, although the above comparisons test the end-member cases.

[29] Here we model an infinitely long vertical strike-slip fault that is embedded in a 50-km-thick elastic plate overlying a viscoelastic half-space with Maxwell time constant $\tau_{m}$. We consider the two cases of deep slip and 


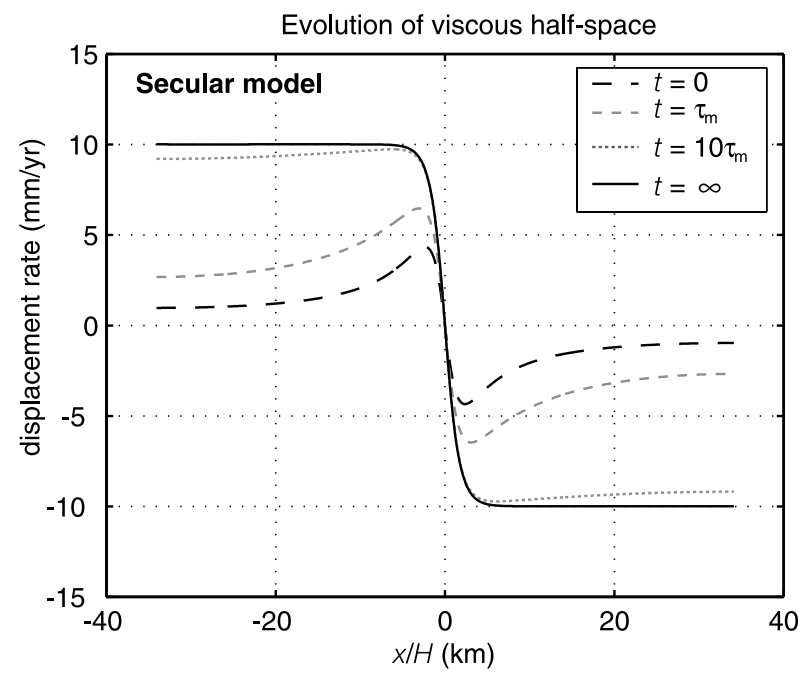

(a)

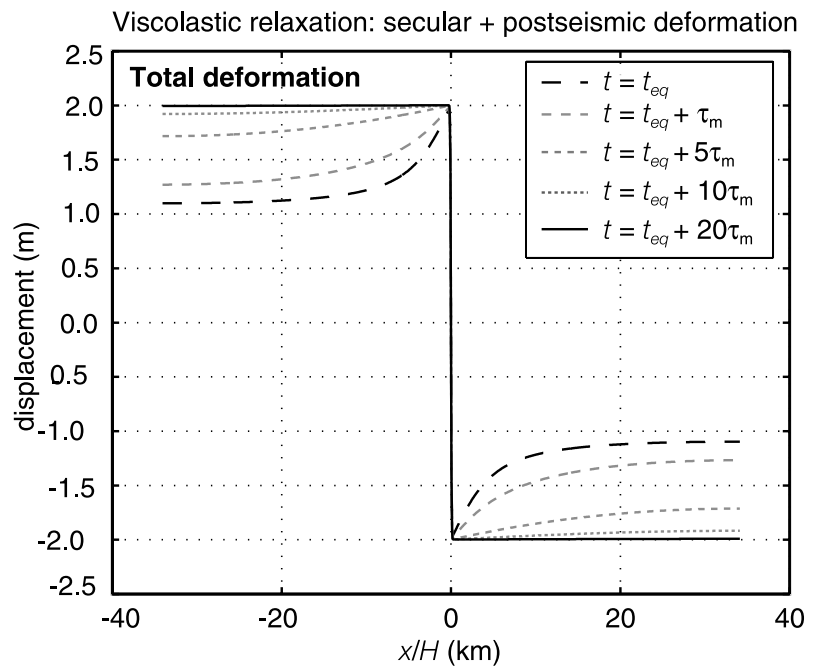

(c)

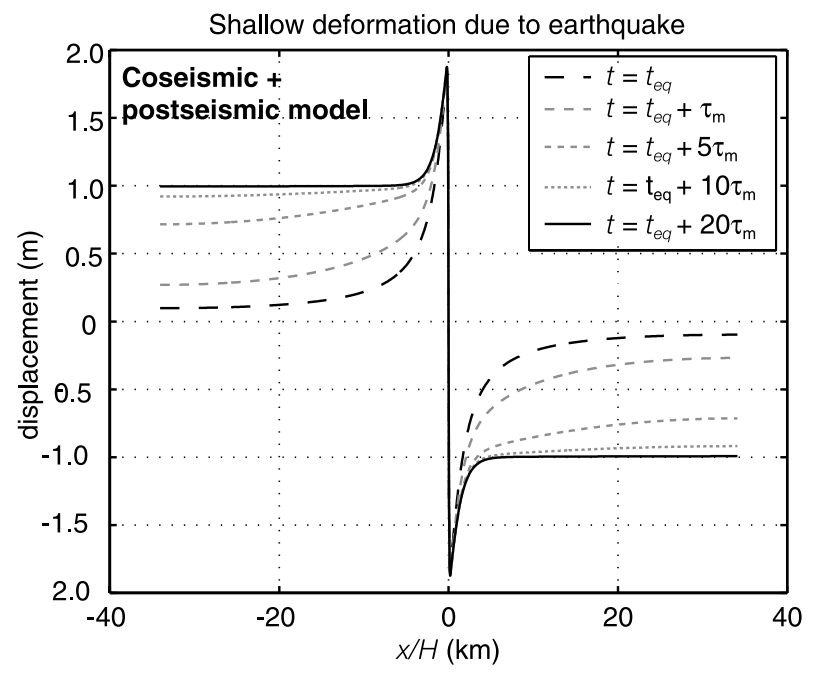

(b)

Figure 3. Fault-parallel displacement profiles of the Fourier model as a function of distance from the fault, $x$, with respect to plate thickness, $H$. Model results are obtained at multiples of Maxwell time $\left(\tau_{m}=\right.$ 24 yrs). Black dashed $(t=0)$ and black solid $(t=\infty)$ profile lines represent time-dependent end-member cases reviewed by Cohen [1999] of the Nur and Mavko [1974] model. (a) Evolution of the secular model showing deep slip over geologic time. Note that the black solid line represents the fully relaxed "secular" model that is used in further models to describe deep slip occurring from the lower depth of the locked fault to the base of the elastic plate. (b) Coseismic (black dashed line) and postseismic models (gray dashed lines) showing shallow deformation from an earthquake occurring at $t_{e q}$ that resulted from $4 \mathrm{~m}$ of accumulated slip. (c) Total deformation resulting from the combination of the secular model $(\mathrm{a}, t=\infty)$ and the time-dependent postseismic models of (b) that capture the full $4 \mathrm{~m}$ of displacement. Note the full block offset of the elastic plate that is illustrated by the step function of (c) for times greater than $20 \tau_{m}$. This behavior is due to deformation contributions from all plate depths (locked and secular).

shallow slip, representing interseismic and coseismic deformation, respectively. In the deep slip case a constant slip rate is applied along the fault in the lower half of the plate (Figure 3a) to simulate interseismic deformation. The initial deformation rate $(t=0)$ matches the elastic half-space solution but eventually evolves to the solution for an elastic plate overlying a fluid half-space $(t=\infty)$. A full step function of plate velocity is achieved at times greater than $t=100 \tau_{m}$, which we will henceforth refer to as the "secular model." In the shallow slip case (Figure $3 b$ ), $4 \mathrm{~m}$ of slip are applied to the upper half of the plate to simulate coseismic and postseismic deformation. The combined displacement (secular model + coseismic/postseismic) (Figure 3c) achieves a full $4 \mathrm{~m}$ step after $20 \tau_{m}$ of postseismic relaxation. This model shows good qualitative agreement with previous studies [e.g., Nur and Mavko, 1974; Rundle and Jackson, 1977; Savage and Prescott, 1978; Ward, 1985; Cohen, 1999].

[30] An important aspect of this "plate" model is that far-field deformation is partitioned into a secular part and 
a seismic part according to the ratio of the lower and upper fault heights [Savage and Prescott, 1978; Ward, 1985]. In this example, where the height ratio of the lower and upper faults is equal, only $1 / 2$ of the far-field deformation is accommodated by the secular model. The other half of the far-field deformation results from repeated earthquakes. While this is an interesting curiosity for purely 2-D models, it has important physical implications for 3-D models. These issues will be discussed in section 4 .

\subsection{Boussinesq Analytic Comparisons}

[31] With the exception of the 3-D elastic half-space solution [Okada, 1985, 1992], thus far we have only discussed 2-D models where the surface normal stress is zero. However, to test our solutions in 3-D requires testing the response of the model to vertical loads (Appendix A). To do this, we first compare our solution to the analytic solution for the response of an elastic half-space to an applied vertical load [Love, 1944]. We then qualitatively examine layered half-space models with and without a gravitational restoring force. Finally, we provide a numerical comparison between our 3-D layered model and the flexure model of a thin elastic plate overlying a fluid halfspace [Brotchie and Silvester, 1969; Turcotte and Schubert, 1982].

\subsubsection{Vertical Point Load on an Elastic Half-Space}

[32] As an initial test, we compare our Boussinesq solution (Appendix A) to the analytic solution for a point load applied to a uniform elastic half-space. The Love [1944] solution for 3-D displacement of an elastic half-space subjected to a point load $(z \neq 0)$ is

$$
\begin{gathered}
U(x, y)=\frac{P}{4 \pi}\left[\frac{1}{\mu} \frac{x z}{r^{3}}-\frac{1}{(\lambda+\mu)} \frac{x}{r(z+r)}\right], \\
V(x, y)=\frac{P}{4 \pi}\left[\frac{1}{\mu} \frac{y z}{r^{3}}-\frac{1}{(\lambda+\mu)} \frac{y}{r(z+r)}\right], \\
W(x, y)=-\frac{P}{4 \pi}\left[\frac{1}{\mu} \frac{z^{2}}{r^{3}}-\frac{(\lambda+2 \mu)}{\mu(\lambda+\mu)} \frac{1}{r}\right],
\end{gathered}
$$

where $U, V$, and $W$ are displacement components as a function of $x, y$, and $z$ spatial coordinates, $P$ is the point load magnitude, $r$ is the radial distance $\left(r^{2}=x^{2}+y^{2}+z^{2}\right)$, and $\lambda$ and $\mu$ are the elastic constants.

[33] For the Fourier model we apply a vertical point load $\left(F_{z}=P=1 \mathrm{MPa}\right)$ to the center of the grid and compare the results at depths of 2 (Figure 4) and 10 grid cell spacings to avoid the singular point in the Love [1944] solutions. The comparison with the analytic solution shows agreement to one part in $10^{2}$ as most of the disagreement occurs directly under the load, which is only two grid cells deep. For these tests, initially we do not include the restoring force of gravity. The code is tested in two ways: first, by equating the elastic constants of the layer and the half-space, and second, by increasing the layer thickness $H$ to 10 times the largest dimension of the grid. These two approaches show agreement to one part in $10^{5}$, which is the accuracy of our single-precision FFT code. (Note that in our computer code the transfer functions are all computed in double precision, but the 2-D arrays are stored in single precision to save computer memory.)

\subsubsection{Gravitational Restoring Force}

[34] We qualitatively investigate the effects of the gravitational restoring force for both half-space and layered models to illustrate that gravity is essential for modeling the long-term behavior of a layered Earth in response to vertical loads. In Appendix A we solve for the Boussinesq coefficients for the following four cases: (1) homogeneous half-space (no gravity); (2) homogeneous half-space with gravity; (3) layered half-space solution (no gravity); and (4) layered half-space solution with gravity. Here we demonstrate the different vertical responses of these special cases due to a vertical point load. In the subsequent examples we use the following parameters: $F_{z}=1 \mathrm{MPa}, H=10 \mathrm{~km}, \mu_{1}=28 \mathrm{GPa}$, and $E_{1}=70 \mathrm{GPa}$. For the two examples that examine the effects of a layered half-space (cases 3 and 4) we use a half-space shear modulus of $\mu_{2}=0$ to simulate an elastic plate overlying a fluid half-space but require the bulk modulus, $\kappa$, to remain constant. In addition, for those examples that include the gravitational contribution (cases 2 and 4), $\rho=3300 \mathrm{~kg} \mathrm{~m}^{-3}$ and $g=9.81 \mathrm{~m} \mathrm{~s}^{-2}$.

\subsubsection{Homogeneous Half-Space Boussinesq Solution} (No Gravity), $\mu_{2}=\mu_{1}, g=0$

[35] This case was discussed in section 3.2.1 and is provided here as a reference model. Figure 5a shows the vertical solution in planform, demonstrating the negative bull's-eye region in the center of the grid. Figure 6 (gray dashed curve) shows the solution in profile.

\subsubsection{Homogeneous Half-Space Boussinesq Solution}

With Gravity, $\mu_{2}=\mu_{1}, g$ Included

[36] Next we include the restoring force of gravity in the homogenous elastic half-space model (Figures 5b and 6, black dashed curve). Note that this solution compares to that of Figure 5a, although magnitudes are slightly larger. It is clear that gravity has little effect on the solution for this model, confirming that gravity can be ignored in elastic half-space dislocation models.

\subsubsection{Layered Half-Space Boussinesq Solution (No Gravity), $\mu_{2}=0, g=0$}

[37] Next we consider the response of a point load applied to an elastic plate overlying a fluid half-space, ignoring the gravitational restoring force (Figure $5 \mathrm{c}$ ). This approach leads to an absurd result with a spatially magnified deflection that is highly dependent upon the dimensions of the grid. This is clearly an unphysical case because the vertical forces are not balanced. The restoring force of gravity is essential in layered dislocation models when the substrate is a fluid.

\subsubsection{Layered Half-Space Boussinesq Solution With} Gravity, $\mu_{2}=0, g$ Included

[38] Lastly, we consider an elastic plate overlying a fluid half-space, this time including the restoring force of gravity (Figures $5 \mathrm{~d}$ and 6, gray solid curve). Including gravity balances the vertical forces and eliminates unreasonable amplitudes, as seen in Figure 5c. Note that the layered model has significantly more vertical deformation than the half-space model.

\subsubsection{Thin Plate Flexure Approximation}

[39] As a partial numerical test of the layered model, we compare our Boussinesq solution to the analytic solution for the flexural response of a thin elastic plate due to a point surface load. The vertical force balance for flexure, $w$, of a 

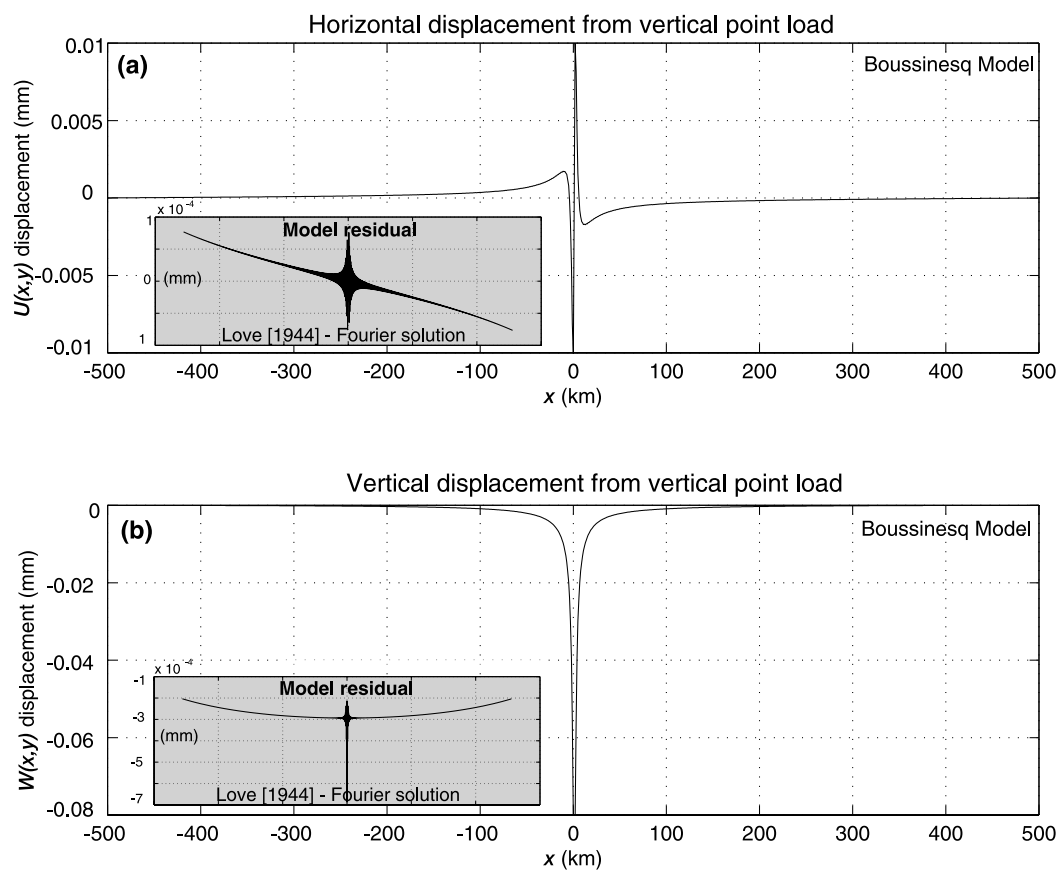

Figure 4. Horizontal and vertical response to a vertical point load applied to a homogeneous elastic medium $\left(\mu_{1}=\mu_{2}\right.$, or $\left.H=\infty\right)$ at a depth of 2 grid cell spacings $(2 \mathrm{~km})$. Boussinesq models are shown for both (a) horizontal $U_{B}$ and (b) vertical $W_{B}$, solutions (from Appendix A). Comparisons with the Love [1944] solutions yield relative errors (gray insets) for both horizontal and vertical components that are primarily less than $1 \%$.

thin elastic plate floating on a fluid half-space [Turcotte and Schubert, 1982] is given by

$$
D \frac{d^{4} w}{d x^{4}}+\rho g w=P(x)
$$

where the vertical load, $P(x)$, is balanced by the flexural resistance of the plate and the gravitational restoring force, $\rho g ; D$ is the flexural rigidity. The flexure solution in the wave number domain is given by

$$
W(k)=P(k)\left[D k^{4}+\rho g\right]^{-1},
$$

where the flexural rigidity is related to the plate thickness and elastic constants $E$ and $v$ (Poisson's ratio) by

$$
D=\frac{E H^{3}}{12\left(1-v^{2}\right)} .
$$

[40] We compare the flexure solution of equation (13) (Figure 5e, Figure 6, solid black curve) to the point load response of the layered Boussinesq solution (Figure 5d). Far from the center of the load, the two models show excellent agreement, although they disagree near the load where the thin plate approximation is no longer valid (Figure 5f). It is interesting to note that the sum of the flexure model (Figure 6, solid black line) and the half-space model (Figure 6, dashed gray line) provides a numerical agreement with the Boussinesq plate solution (Figure 6, solid gray line) to an accuracy of $0.1 \%$. These results are also confirmed by the static flexure solution of Brotchie and Silvester [1969] with similar parameters.

\section{3-D Time-Dependent Deformation and Stress}

[41] Having demonstrated the 2-D behavior and accuracy of our Fourier model, along with the vertical response of the layered Boussinesq solution, we now present a 3-D simulation of the earthquake cycle that includes multiple fault elements and explores postseismic deformation for intermediate timescales following an earthquake. The basic model (Figure 7) consists of a fault with three independent segments, A, B, and C, that are embedded in a 50-km-thick elastic plate that is loaded by $40 \mathrm{~mm} \mathrm{yr}^{-1}$ of strike-slip plate motion. Between earthquakes the middle fault segment, B, is locked from the surface to a depth of $25 \mathrm{~km}$, below which deep, secular slip occurs. The two adjacent fault segments, $\mathrm{A}$ and $\mathrm{C}$, are allowed to slip completely to the surface, simulating uniform fault creep. In this model the fault system is a mature one (geologically evolved), where although $t=0$ years represents the time of model initiation, we assume that a full secular velocity plate step is already in place. The model spans $300+$ years, where the first 100 years include secular tectonic loading. At $t=100$ years we simulate an earthquake by initiating $4 \mathrm{~m}$ of coseismic shallow slip (depths $<25 \mathrm{~km}$ ) on segment B. Postseismic deformation, due to viscoelastic relaxation of the half-space, begins immediately after the event. We present single-yearaveraged snapshots at multiples of Maxwell time for both $3-\mathrm{D}$ velocity and Coulomb stress. Animated movies of 

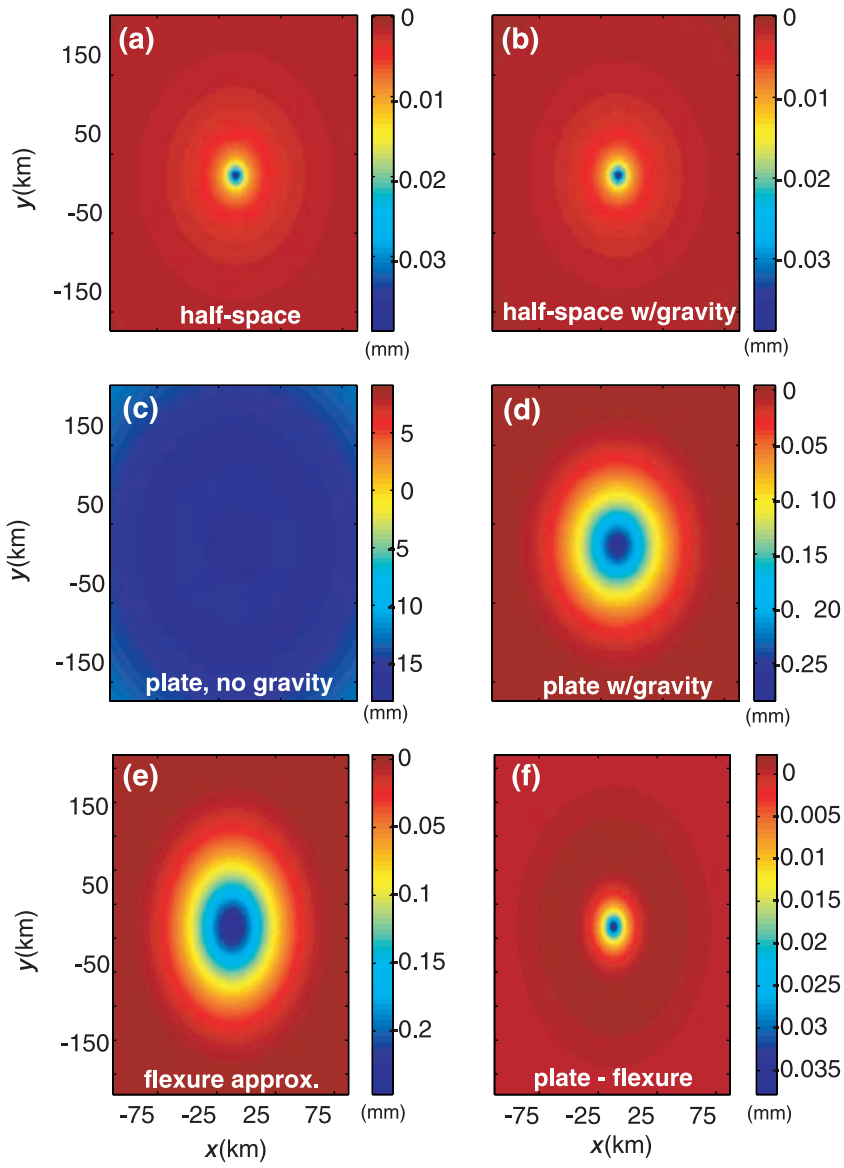

Figure 5. Map view of the vertical Boussinesq response (in $\mathrm{mm}$ ) to a vertical point load: (a) homogeneous elastic half-space model without a gravitational restoring force; (b) homogeneous elastic half-space model with a gravitational restoring force; (c) elastic plate overlying a fluid half-space model without a gravitational restoring force; (d) elastic plate overlying a fluid half-space model with a gravitational restoring force; (e) flexural response from the thin plate approximation [Le Pinchon et al., 1973; McKenzie and Bowin, 1976]; (f) residual difference between (d) and (e). Note that color scale differs for each plot.

these models can be found online at http://topex.ucsd.edu/ body_force.

\subsection{3-D Velocity}

[42] The 3-D velocity field is computed by calculating the change in displacement over 1 year time increments. For secular velocity the time increment is largely irrelevant as the secular model behavior is assumed steady state. However, when an earthquake occurs during the time interval, the velocity is equal to the coseismic/postseismic deformation divided by 1 year. Secular velocity is shown in map view (Figure 8a), where $U$ represents faultperpendicular velocity, $V$ represents fault-parallel velocity, and $W$ represents vertical velocity. The fault-parallel velocity $V$ shows a step change across the creeping segments (A and $\mathrm{C}$ ) and a more gradual transition across the locked section. Alternatively, the $U$ and $W$ components show little velocity contribution from the segments that are creeping, while moderate amplitudes, $10 \mathrm{~mm} \mathrm{yr}^{-1}$ and

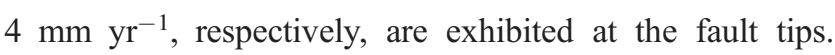
Also noted are the reversing quadrants of the vertical velocity field, $W$, and also the reversal of amplitude in the near and far fields. For example, in the far field, positive velocity (uplift) is noted in the direction of fault movement, while in the near field, negative velocity (subsidence) is found at the fault tip. The 3-D secular behavior of the earthquake cycle demonstrated here is assumed to be steady state.

[43] Coseismic velocity is shown in Figure 8b. Both $U$ and $W$ velocities reverse sign and reach amplitudes of $\pm 0.8 \mathrm{~m} \mathrm{yr}^{-1}$ and $\pm 0.2 \mathrm{~m} \mathrm{yr}^{-1}$, respectively, in response to the earthquake. Alternatively, the fault-parallel velocity component, $V$, coseismically responds by lurching forward in the direction of tectonic motion at $\pm 2 \mathrm{~m} \mathrm{yr}^{-1}$ at the time of the earthquake. This type of behavior has been established by previous fault models [e.g., Chinnery, 1961; Okada, 1985, 1992; Yang and Toksoz, 1981] for elastic strike-slip deformation of a vertical fault.

[44] Deformation continues for several Maxwell times (Figures 9-11) following the earthquake. Note that we have removed the secular (tectonic loading) component for times following the earthquake in order to isolate the postseismic velocity response. The $U$ component (Figure 9) is reversed in sign with respect to the secular model and slowly diminishes in both wavelength and magnitude, completely dissipating by $\sim 10 \tau_{m}$, or $\sim 240$ years, after the earthquake. Likewise, the $V$ component (Figure 10) slowly decreases in magnitude and spatial dimension before completely disappearing by $\sim 10 \tau_{m}$. Finally, the vertical velocity component, $W$ (Figure 11), demonstrates an accelerative behavior for a short time after the earthquake. The vertical velocity field increases for times less than $2 \tau_{m}$, followed by a slow decrease that remains for times greater than $5 \tau_{m}$. The timescale for such an acceleration/deceleration of deformation direction depends strongly on plate thickness and halfspace density. Like the other components, the vertical velocity diminishes completely by $10 \tau_{m}$ following the earthquake.

\subsection{Coulomb Stress}

[45] Coulomb stress provides a measure of the shear loading on faults of a particular azimuth [e.g., Stein et al., 1994; Harris, 1998; Harris and Simpson, 1998], where positive Coulomb stress indicates that a fault plane is brought closer to failure, while negative stress indicates that the fault plane has moved away from failure. To calculate Coulomb stress, we follow the approach of King et al. [1994], where the Coulomb failure criterion, $\sigma_{f}$, is defined by

$$
\sigma_{f}=\tau-\mu_{f} \sigma_{n}
$$

In this equation, $\sigma_{n}$ and $\tau$ are the normal and shear stress on a failure plane, respectively, and $\mu_{f}$ is the effective coefficient of friction. Our model provides the 3-D vector displacement field from which we compute the stress tensor. Right-lateral shear stress and extension are assumed to be positive. Because Coulomb stress is zero at the surface and becomes singular at the locking depth, we calculate Coulomb stress at $1 / 2$ of the local locking depth [King et al., 1994] and choose $\mu_{f}$ to be 0.6 . 


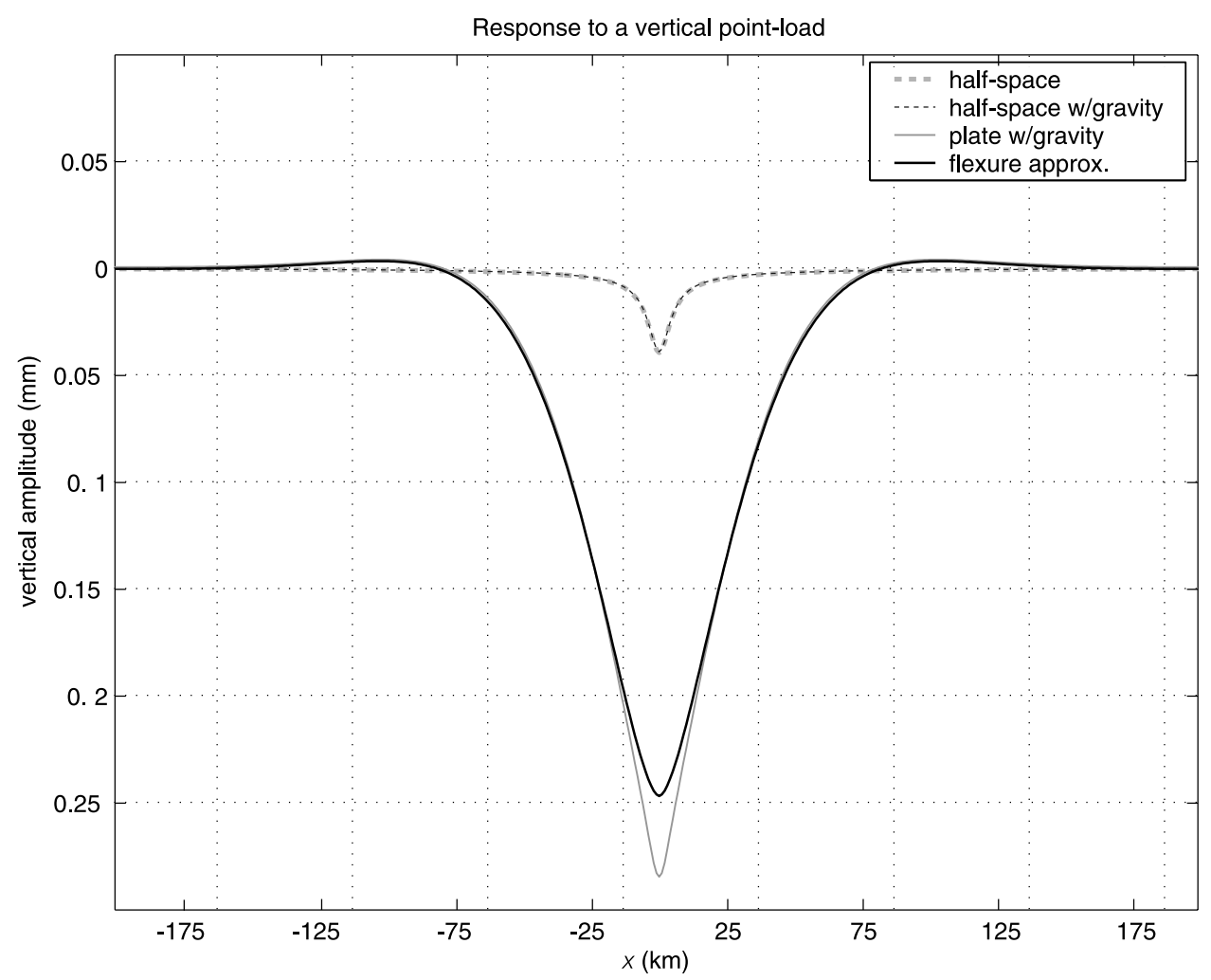

Figure 6. Vertical profiles as a function of distance, $x$, acquired for the Boussinesq results of Figure 5 for a half-space model without a gravitational restoring force (dashed gray line, a), a half-space model with a gravitational restoring force (dashed black line, b), an elastic plate overlying a fluid half-space model (solid gray line, d), and the thin plate flexure approximation (solid black line, e). Combining the vertical results of the half-space model (dashed gray line) and the flexure solution (solid black line) yields a model with numerical accuracy of $10^{-3}$ when compared to the Boussinesq plate model (solid gray line).

[46] Our objective is to track the accumulation of Coulomb stress both before and after an earthquake (Figure 12). During the 100 years prior to the earthquake, Coulomb stress accumulates near the locked fault at a rate of $\sim 0.04 \mathrm{MPa} \mathrm{yr}^{-1}$, ultimately increasing to a peak value of $4 \mathrm{MPa}$ (Figure 12, $t=25,50,75$ years). An earthquake releases all accumulated stress and even reverses the sign. In order to isolate the postseismic effects due to viscoelastic relaxation, we have removed the secular (tectonic loading) component for times greater than $t=100$ years. A zone of negative Coulomb stress (stress shadow) develops and then decreases in amplitude and wavelength for at least $2 \tau_{m}$, or $\sim 50$ years. Had we included the secular tectonic component, the Coulomb stress shadow would have existed for only $\sim 0.5 \tau_{m}$, or $\sim 13$ years, followed by a repeated stress accumulation process.

\section{Discussion}

[47] These examples demonstrate essential features of 3-D deformation and stress during the earthquake cycle and agree with other full 3-D numerical models of the earthquake cycle [Deng et al., 1998; Kenner and Segall, 1999; Pollitz et al., 2000, 2001; Zeng, 2001]. The primary difference between a layered viscoelastic model and an elastic half-space model is in the vertical velocity.

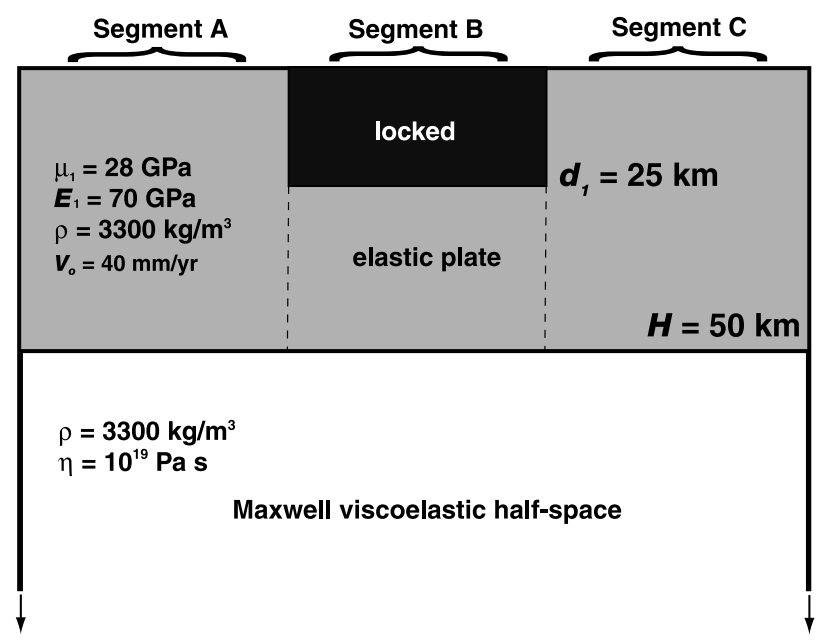

Figure 7. Along-fault vertical transect of the threesegment model embedded in an elastic plate of $50 \mathrm{~km}$ overlying a Maxwell viscoelastic half-space. Segments A and $\mathrm{C}$ are identified by zero locking depths (creeping), while segment B is locked from the surface $\left(d_{2}=0\right)$ to a depth of $25 \mathrm{~km}$. Model parameters included shear modulus $\left(\mu_{1}\right)$, Young's modulus $\left(E_{1}\right)$, density $(\rho)$, and half-space viscosity ( ). Secular plate velocity, $V_{o}$, is set to $40 \mathrm{~mm} \mathrm{yr}^{-1}$. 


\section{(a) secular velocity}
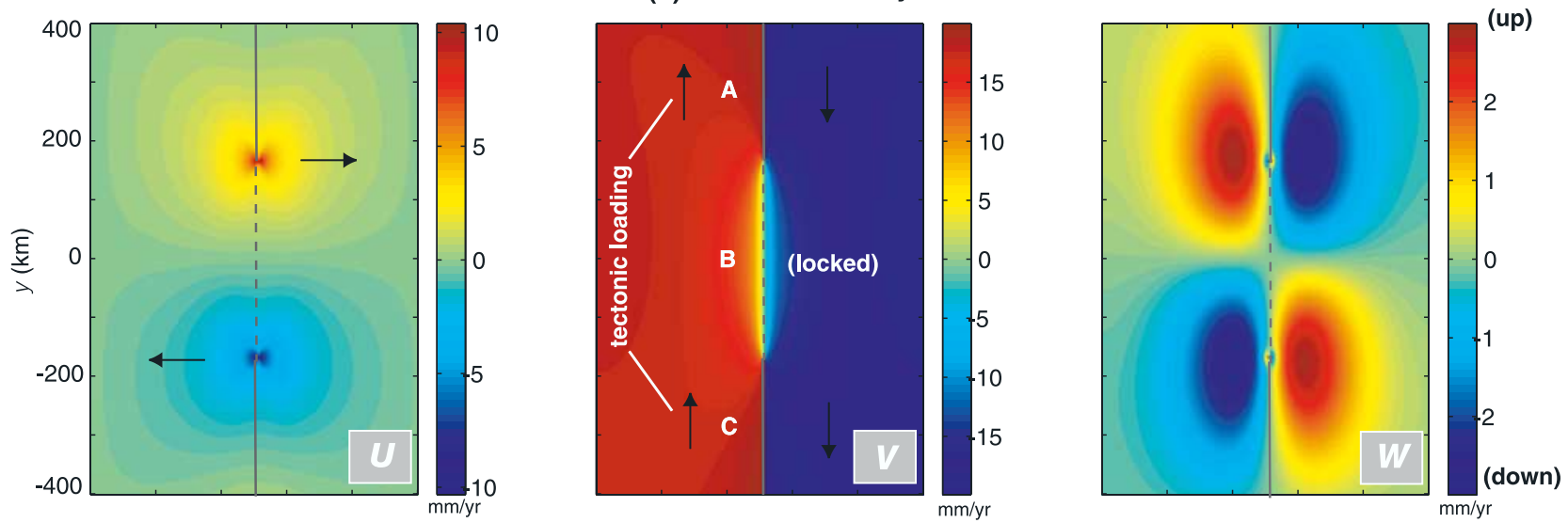

(b) coseismic velocity

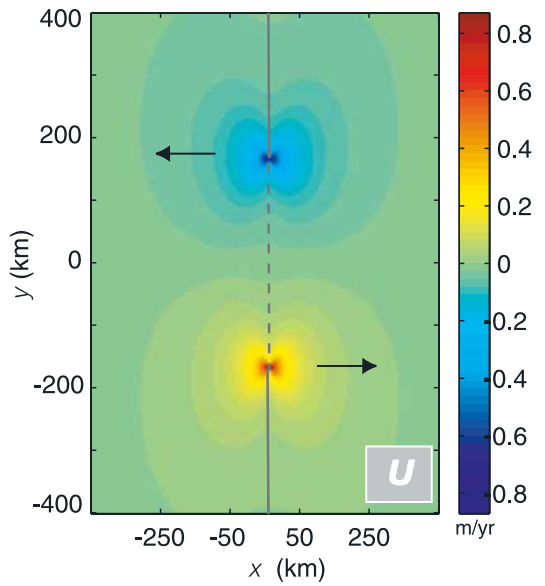

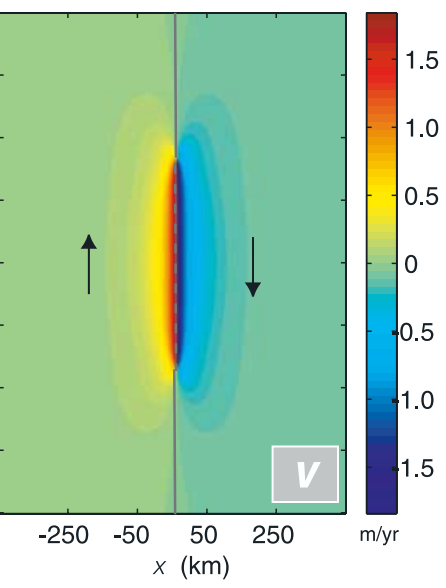

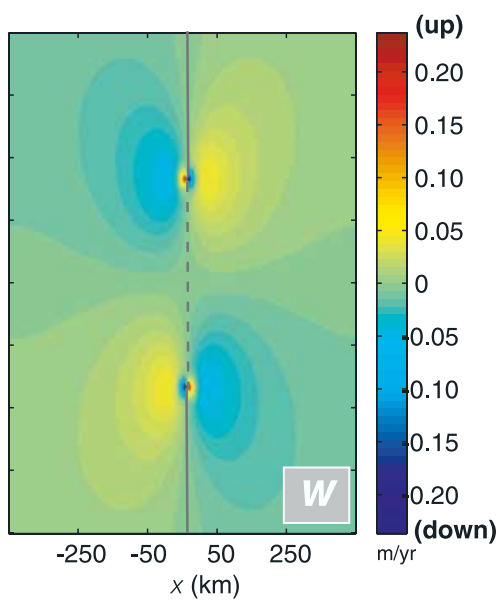

Figure 8. Map view of velocity results of the three-segment model (segments $A, B$, and C) shown for $U$ (fault-perpendicular), $V$ (fault-parallel), and $W$ (vertical) components for (a) secular velocity $(\mathrm{mm} / \mathrm{yr}$ ) and (b) coseismic velocity (or displacement difference over one year, $\mathrm{m} / \mathrm{yr}$ ) from an earthquake event at $t=t_{e q}=100$ years. Because the secular behavior of our model is assumed to be steady state, (a) represents the 3-D velocity field for times $t=0-99$ years. Creeping segments A and $\mathrm{C}$ are identified by a solid gray line, while locked fault segment $\mathrm{B}$ is indicated by a gray dashed line.

Following a right-lateral earthquake, we observe increasing vertical velocity (Figure 11) that produces uplift in the northeast and southwest quadrants and subsidence in the northwest and southeast quadrants. This behavior persists for at least two Maxwell times ( $\sim 50$ years) and then gradually subsides.

[48] The wavelength and timescale of this vertical velocity feature is largely dependent upon the elastic plate thickness and half-space viscosity. The wavelength of vertical deformation is related to the flexural wavelength, and thus for a $50-\mathrm{km}$-thick plate the characteristic wavelength of the vertical deformation pattern is $\sim 440 \mathrm{~km}$. However, the observed lobate vertical velocity patterns following the Landers and Hector Mine earthquakes have a much smaller horizontal wavelength, requiring a much thinner plate $(\sim 10 \mathrm{~km})$ [Deng et al., 1998; Pollitz et al., 2001].

[49] The timescale of the vertical velocity acceleration and decay also depends on both the plate thickness and the half-space viscosity. A plate of $25 \mathrm{~km}$ thickness, as opposed to a 50-km-thick plate, responds on a longer timescale, suggesting that the postglacial rebound timescale is providing a minor contribution to the vertical response. The postglacial rebound timescale [Turcotte and Schubert, 1982] is given by

$$
\tau_{g}=4 \pi \eta / \rho g \lambda,
$$

where $\lambda$ is the wavelength of deformation and $\eta, \rho$, and $g$ are viscosity, density, and gravity, respectively. Because rebound timescale is inversely proportional to the wavelength of the deformation (which is controlled by the flexural wavelength of the elastic plate), a thinner plate (i.e., smaller $\lambda$ ) has a longer postglacial rebound timescale. The $50-\mathrm{km}$-thick plate has a postglacial timescale of 280 years for a viscosity of $10^{19} \mathrm{~Pa} \mathrm{~s}$, while the shorter wavelength associated with the thinner plate has a longer postglacial timescale (500 years).

[50] Our model also demonstrates stress behavior due to time-dependent postseismic readjustment (Figure 12) in 

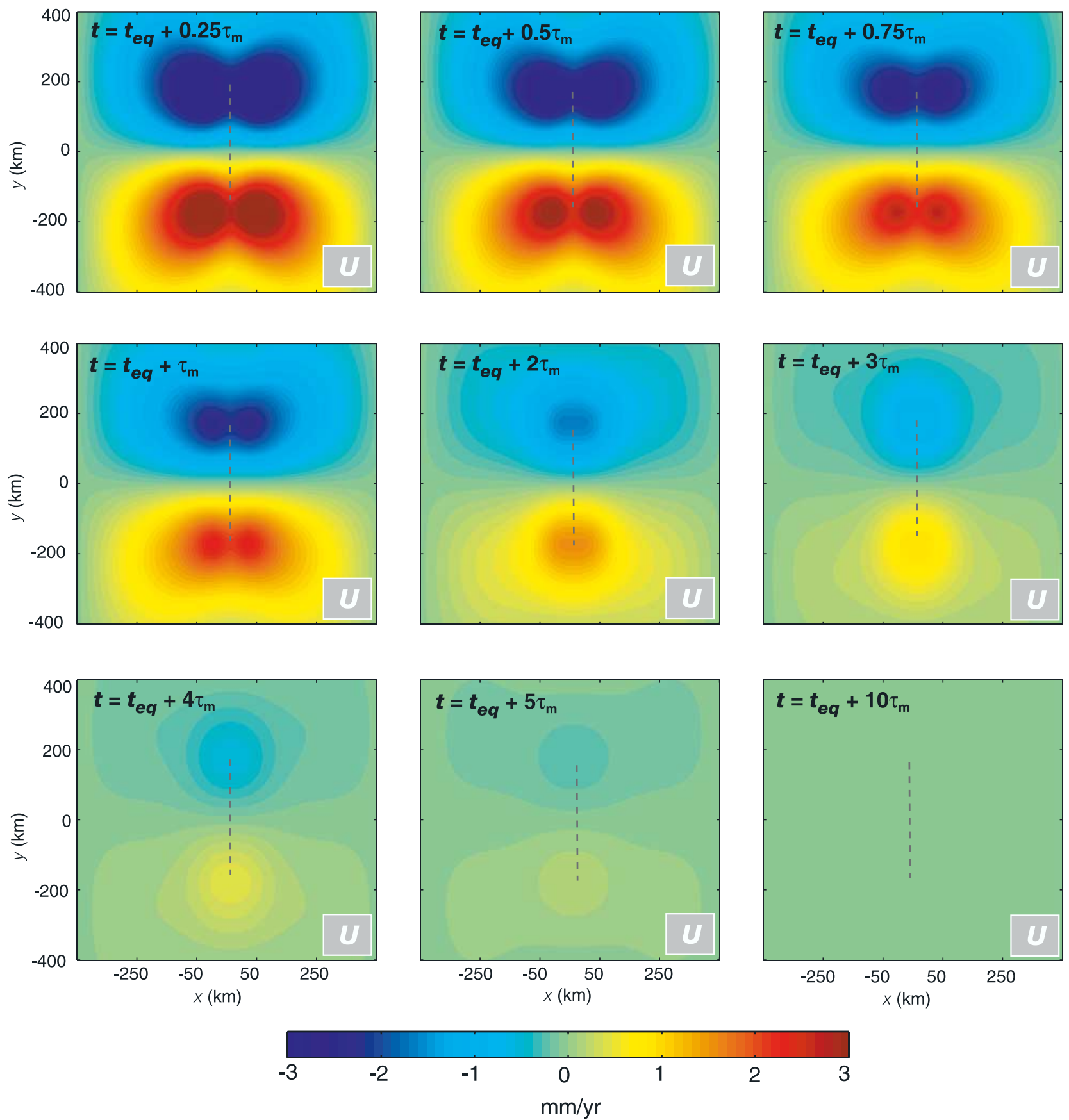

Figure 9. Map view of postseismic response for $U$ (fault-perpendicular) horizontal velocity component at $\mathrm{t}=100 \mathrm{yrs}\left(t_{e q}\right)+$ multiples of Maxwell time $\left(0.25,0.5,0.75,1,2,3,4,5\right.$, and $\left.10 \tau_{\mathrm{m}}\right) \mathrm{in} \mathrm{mm} / \mathrm{yr}$. Displacement is calculated at half-year increments and velocity is computed by differencing two of these increments spanning one year. The gray dashed line indicates location of fault segment B. Positive velocities indicate change in displacement in the positive $x$ direction; negative velocities indicate change in displacement in the negative $x$ direction. The velocity for the $U$ component slowly decreases after the earthquake and diminishes completely by approximately $10 \tau_{\mathrm{m}}$.

agreement with previous studies [Harris and Simpson, 1993, 1996; Zeng, 2001]. Following an earthquake, a stress shadow develops. This stress shadow should, in theory, inhibit the occurrence of subsequent seismic events such as aftershocks and large triggered earthquakes. As time advances, the spatial extent and magnitude of the stress shadow decays nonuniformly [Ward, 1985]. Eventually the locked fault becomes reloaded with tectonic stress and relaxation ceases, resulting in positive stress accumulation surrounding the fault and a resumption of the earthquake cycle.

[51] Realistic models exhibiting similar stress shadowing behaviors and fault interactions have been explored by other workers [e.g., Kenner and Segall, 1999; Parsons, 2002]. A 2-D postseismic shear stress model of Kenner and Segall [1999] demonstrated that an initial stress 

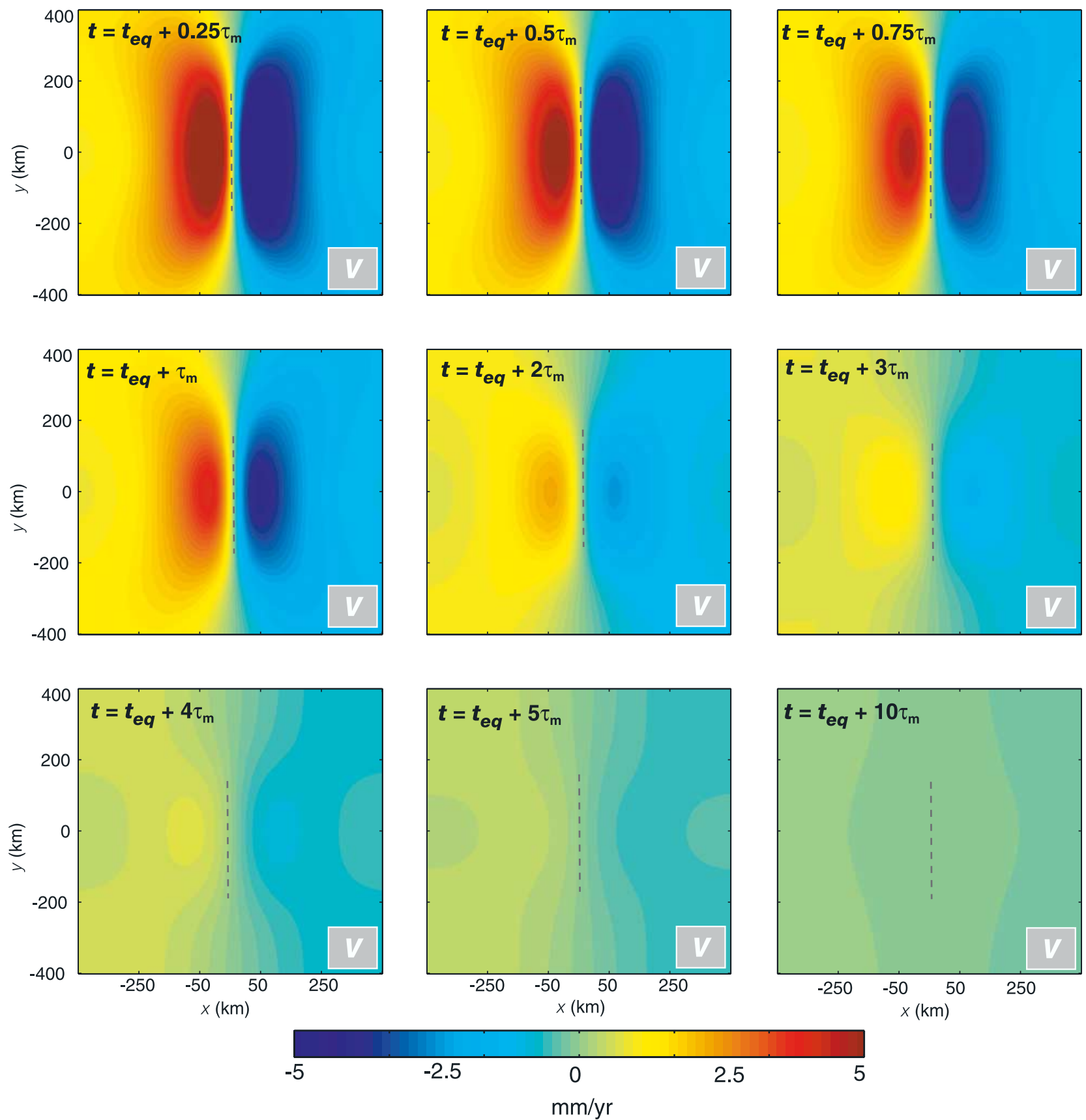

Figure 10. Map view of postseismic response for $V$ (fault-parallel) horizontal velocity component at $t=100 \mathrm{yrs}\left(t_{e q}\right)+$ multiples of Maxwell time $\left(0.25,0.5,0.75,1,2,3,4,5\right.$, and $\left.10 \tau_{m}\right) \mathrm{in} \mathrm{mm} / \mathrm{yr}$. Positive velocities indicate change in displacement in the positive $y$ direction; negative velocities indicate change in displacement in the negative $y$ direction. The velocity for the $V$ component slowly decreases after the earthquake and diminishes completely by approximately $10 \tau_{\mathrm{m}}$.

decrease, followed by viscoelastic relaxation, encouraged increases of stress duration and magnitude for particular fault geometries. In addition, changes in Coulomb stress are shown to be highly sensitive to kinks in fault geometry and jumps in slip distribution [Freed and Lin, 2001; Kilb et al., 2002]. While we have obviously eliminated such geometrical effects by embedding a straight fault system of constant slip with depth for this analysis, more complicated simulations have shown high rates of stress at junctions of fault bends. A fault system with bends and kinks produces anomalous Coulomb stress at fault segment tips that is never fully released by strikeslip motion. Alternative mechanisms, such as normal faulting, may be required to cancel accumulating stress due to geometrical effects. These ideas will be more completely explored in a following paper (B. Smith and D. Sandwell, manuscript in preparation, 2004).

\section{Conclusions}

[52] We have developed and tested a semianalytic model for the 3-D response of a Maxwell viscoelastic 

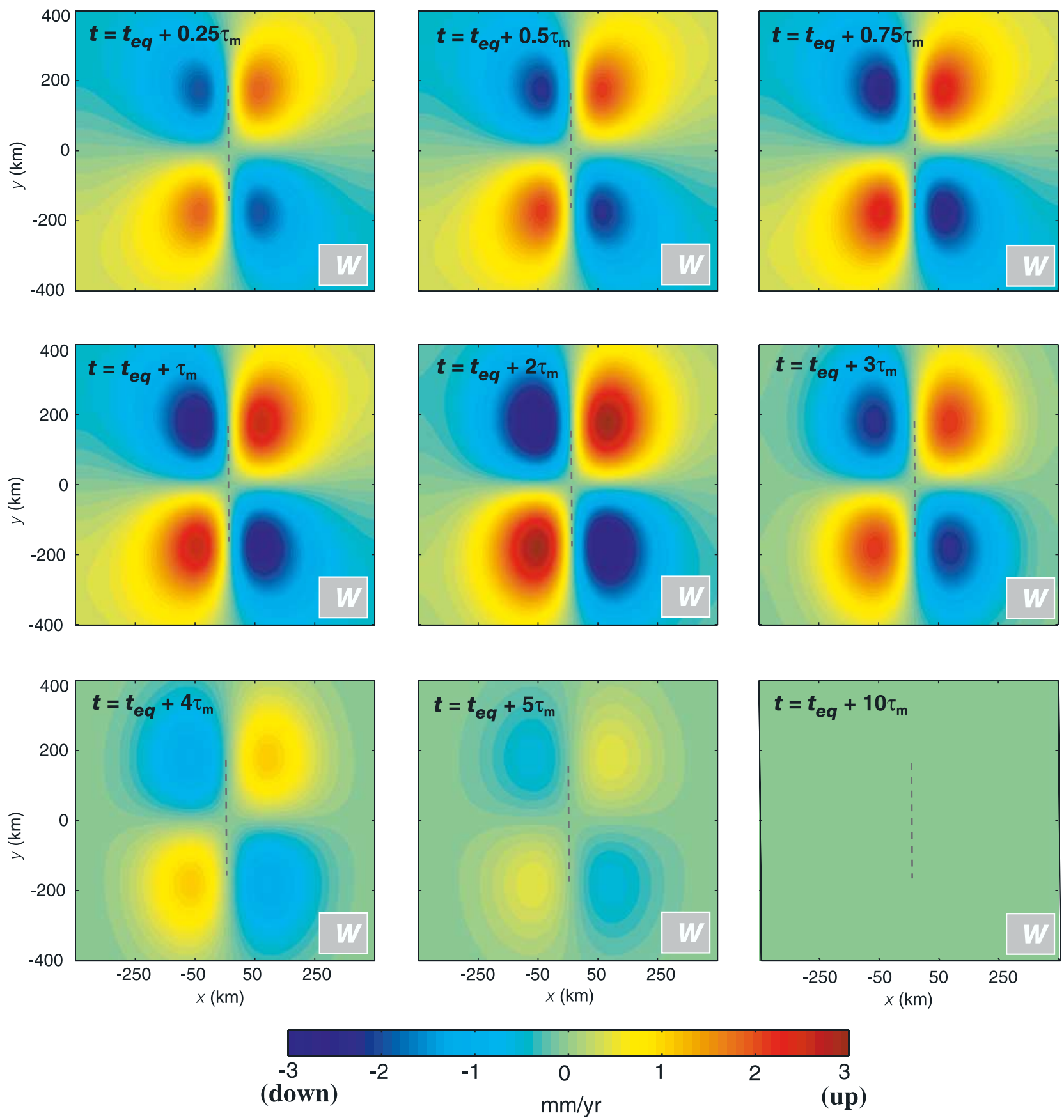

Figure 11. Map view of postseismic response for $W$ (vertical) velocity component at $t=100 \mathrm{yrs}\left(t_{\text {eq }}\right)+$ multiples of Maxwell time $\left(0.25,0.5,0.75,1,2,3,4,5\right.$, and $\left.10 \tau_{m}\right)$ in $\mathrm{mm} / \mathrm{yr}$. Positive velocities indicate change in displacement in the positive $z$ direction (uplift); negative velocities indicate change in displacement in the negative $z$ direction (subsidence). The velocity for the $W$ component temporarily increases after the earthquake until approximately $2 \tau_{\mathrm{m}}$, followed by a decrease in velocity in the same directional sense.

layered half-space due to an arbitrary distribution of body forces. For a vertical fault, 2-D convolutions are performed in the Fourier transform domain, and thus displacement, strain, and stress due to a complicated fault trace can be computed very quickly. Using the Correspondence Principle, the solutions for a layered elastic half-space are easily extended to that of a viscoelastic half-space without increasing the computational burden.
The horizontal complexity of the fault system has no effect on the speed of the computation; a model with a prescribed time, consisting of hundreds of fault elements, requires less than $40 \mathrm{~s}$ of CPU time on a desktop computer. Because multiple earthquakes are required to fully capture viscoelastic behavior, our model is capable of efficiently computing 3-D viscoelastic models spanning thousands of years. 

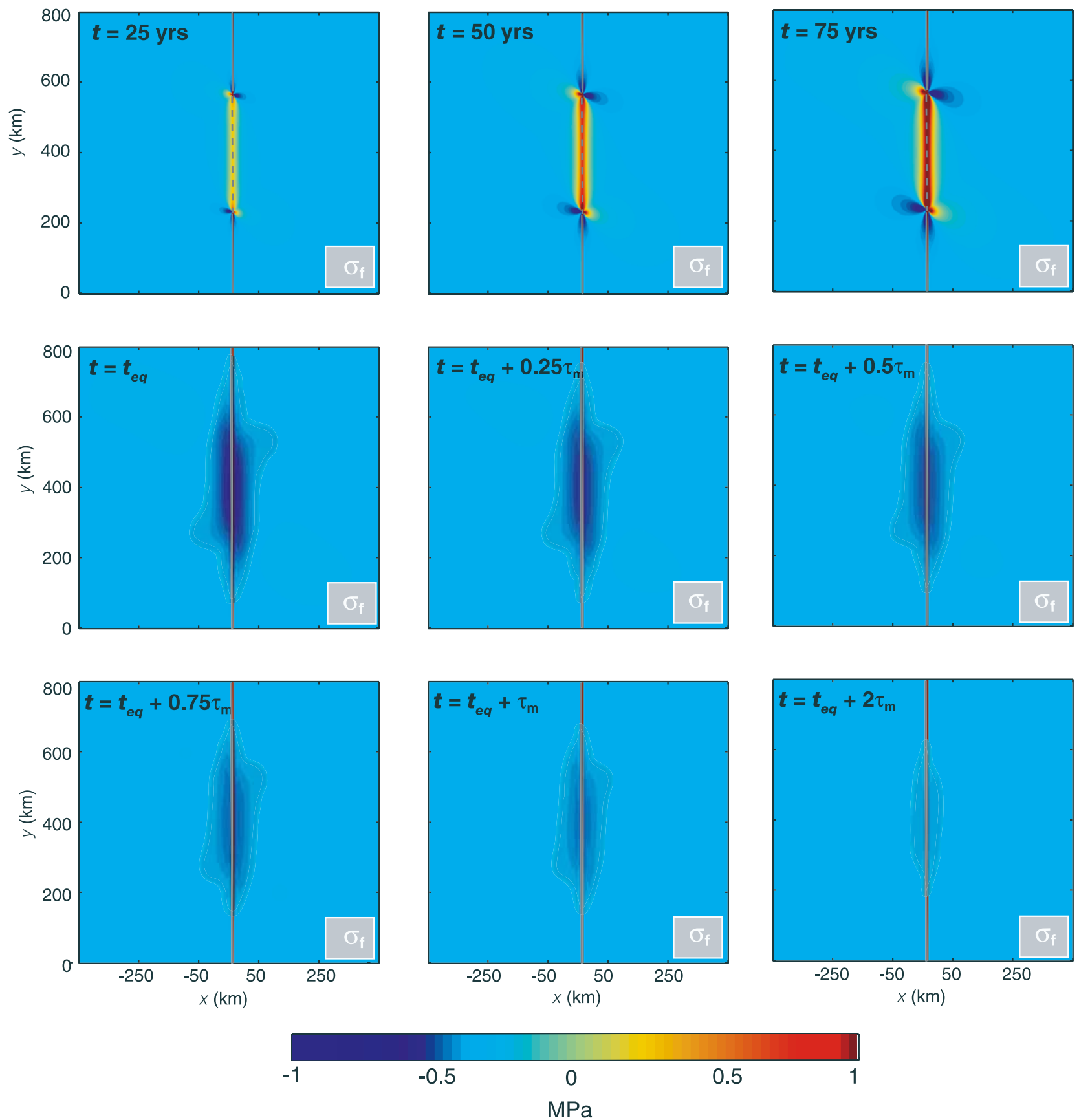

Figure 12. Map view results of Coulomb stress in MPa for a typical earthquake cycle. For the first 100 years $(t=25,50,75, \ldots$ yrs $)$, secular Coulomb stress accumulates on the locked fault at a rate of $\sim 0.04 \mathrm{MPa} /$ year. At $t=t_{e q}=100 \mathrm{yrs}$, an earthquake occurs that removes all of the positive stress, followed by snapshots of the Coulomb stress shadow $\left(t=100^{+}\right.$yrs $)$marked by regions of negative stress. In order to isolate the postseismic effects due to stress transfer, we have removed the secular (tectonic loading) component for times greater than $t=100 \mathrm{yrs}$.

[53] Our model has the accuracy and speed necessary for computing both geometrically and temporally complex models of the earthquake cycle. Here we have demonstrated the basic 2-D and 3-D deformation behavior of a generalized simple fault system. We find that the evolving velocity field, particularly the vertical component, demonstrates an overall decelerative behavior dependent upon plate thickness, although a temporary velocity increase is also observed. We also investigate the temporal behavior of the Coulomb stress field and find that a stress shadow exists for at least two Maxwell times and slowly decays as stress is redistributed in the plate and tectonic loading dominates.

[54] Now that this modeling approach is understood and fully tested, it will be used to simulate the complex timedependent stress evolution of realistic tectonic boundaries 
on Earth, such as the San Andreas Fault system. We are currently establishing a suite of models, consistent with both geodetic and geological observations, that will increase our understanding of how temporal plate boundary deformation and stress variations within the seismogenic crust can result from different tectonic settings throughout the earthquake cycle.

\section{Appendix A: Boussinesq Problem for a Layered Half-Space}

[55] The Boussinesq problem [Boussinesq, 1885; Steketee, 1958] offers a supplemental solution for removing anomalous vertical normal stress that arises from the method of images [Weertman, 1964], used to partially satisfy the zero-shear surface boundary condition for a homogeneous elastic half-space. Likewise, for a layered elastic halfspace, multiple image sources must be included to partially satisfy the surface boundary condition (Appendix B) and the Boussinesq approach must be used to remove the remaining normal tractions. Unfortunately, the Boussinesq solution for a homogeneous half-space does not fully satisfy the boundary conditions for a layered elastic halfspace and an alternative solution must be derived. Our contribution to this problem is to develop a Boussinesqlike solution that reflects a new set of elastic solutions that accounts for normal tractions in a layer overlying a half-space.

[56] When solving the Boussinesq problem for a layered elastic half-space, an approach similar to that of the homogeneous half-space applies [e.g., Steketee, 1958; Smith and Sandwell, 2003]. The major exceptions to this approach are the additional boundary conditions [Burmister, 1943] (note that the following notation uses subscripts 1 and 2 to refer to layer and half-space displacement and stress, respectively, and a lowercase $h$ is used in place of $H$ for the plate thickness, or layer interface):

[57] 1. The surface layer must be free of shear and normal stress, except those imposed to balance the remaining normal tractions $\left(\tau_{33}\right)$ and provide gravitational support $(\rho g)$, if necessary. Gravity plays an important role in modulating long-term vertical motion:

$$
\tau_{z z 1}=-\tau_{33}+\left.\rho g W_{1}\right|_{z=0} \quad \tau_{x z 1}=\tau_{y z 1}=\left.0\right|_{z=0} .
$$

[58] 2. Stress and displacement across the layer interface must be continuous:

$$
\begin{array}{cc}
\tau_{x z 1}=\left.\tau_{x z 2}\right|_{z=-h} \quad U_{1}=\left.U_{2}\right|_{z=-h} \\
\tau_{y z 1}=\left.\tau_{y z 2}\right|_{z=-h} \quad V_{1}=\left.V_{2}\right|_{z=-h} . \\
\tau_{z z 1}=\left.\tau_{z z 2}\right|_{z=-h} \quad W_{1}=\left.W_{2}\right|_{z=-h}
\end{array}
$$

[59] 3. At infinite depth, stress and displacements below the layer must go to zero:

$$
\tau_{x z 2}=\tau_{y z 2}=\tau_{z z 2}=\left.0\right|_{z=-\infty} \quad U_{2}=V_{2}=W_{2}=\left.0\right|_{z=-\infty} .
$$

[60] Our approach is to find the Galerkin vector $\Gamma_{i}$ [Steketee, 1958] for the complimentary solutions that satisfy the above boundary conditions for both displacements and stress in both the elastic layer (layer 1) and the half-space below (layer 2). We begin by writing both displacement and stress in terms of the Galerkin vector:

$$
\begin{gathered}
u_{i}=\Gamma_{i, k k}-\alpha \Gamma_{k, k i} \\
\tau_{i j}=\lambda(1-\alpha) \delta_{i j} \Gamma_{k, k l l}+\mu\left(\Gamma_{j, i k k}+\Gamma_{i, j k k}\right)-2 \mu \alpha \Gamma_{k, k i j},
\end{gathered}
$$

or, more explicitly,

$$
\begin{array}{cc}
U_{B}=-\alpha \frac{\partial^{2} \Gamma}{\partial x \partial z}, & \tau_{x z}=\mu \frac{\partial}{\partial x}\left\{\nabla^{2} \Gamma-2 \alpha \frac{\partial^{2} \Gamma}{\partial z^{2}}\right\}, \\
V_{B}=-\alpha \frac{\partial^{2} \Gamma}{\partial y \partial z}, & \tau_{y z}=\mu \frac{\partial}{\partial y}\left\{\nabla^{2} \Gamma-2 \alpha \frac{\partial^{2} \Gamma}{\partial z^{2}}\right\}, \\
W_{B}=-\alpha \frac{\partial^{2} \Gamma}{\partial z^{2}}+\nabla^{2} \Gamma, & \tau_{z z}=\mu \frac{\partial}{\partial z}\left\{\left(\frac{\alpha}{\xi}\right) \nabla^{2} \Gamma-2 \alpha \frac{\partial^{2} \Gamma}{\partial z^{2}}\right\},
\end{array}
$$

where $\mu$ and $\lambda$ are the elastic constants, $\alpha=(\lambda+\mu) /(\lambda+2 \mu)$, and $\xi=(\lambda+\mu) /(3 \lambda+4 \mu)$.

[61] As described by Love [1929] and Timoshenko [1934], the stress and displacement equations of elasticity must satisfy the equation of compatibility, most commonly known as the biharmonic equation:

$$
\nabla^{4} \Gamma=\frac{\partial}{\partial x^{2}}\left(\nabla^{2} \Gamma\right)+\frac{\partial}{\partial y^{2}}\left(\nabla^{2} \Gamma\right)+\frac{\partial}{\partial z^{2}}\left(\nabla^{2} \Gamma\right)=0 .
$$

The general solution to this problem is

$$
\Gamma=(A+C z) e^{\beta z}-(B+D z) e^{-\beta z},
$$

where $\beta=2 \pi|\mathbf{k}|$ and $|\mathbf{k}|=k_{x}^{2}+k_{y}^{2}$. For the layered model, layers 1 and 2 will have the following representation with corresponding elastic constants $\mu_{1}, \lambda_{1}$, and $\mu_{2}, \lambda_{2}$ :

$$
\begin{aligned}
& \Gamma_{1}=\left(A_{1}+C_{1} z\right) e^{\beta z}-\left(B_{1}+D_{1} z\right) e^{-\beta z} \\
& \Gamma_{2}=\left(A_{2}+C_{2} z\right) e^{\beta z}-\left(B_{2}+D_{2} z\right) e^{-\beta z} .
\end{aligned}
$$

Likewise, the two layers will have displacements and stress as functions of their respective Galerkin vectors:

$$
\begin{gathered}
\text { layer } 1=U_{B 1}\left(\Gamma_{1}\right), V_{B 1}\left(\Gamma_{1}\right), W_{B 1}\left(\Gamma_{1}\right), \tau_{\mathrm{xz} 1}\left(\Gamma_{1}\right), \\
\tau_{\mathrm{yz} 1}\left(\Gamma_{1}\right), \tau_{\mathrm{zz} 1}\left(\Gamma_{1}\right) \\
\text { layer } 2=U_{B 2}\left(\Gamma_{2}\right), V_{B 2}\left(\Gamma_{2}\right), W_{B 2}\left(\Gamma_{2}\right), \tau_{\mathrm{xz} 2}\left(\Gamma_{2}\right), \\
\tau_{\mathrm{yz} 2}\left(\Gamma_{2}\right), \tau_{\mathrm{zz} 2}\left(\Gamma_{2}\right)
\end{gathered}
$$

[62] To satisfy the zero displacement boundary condition as $z \rightarrow-\infty, B_{2}=D_{2}=0$. The Galerkin vectors (A8) then take on the form

$$
\begin{gathered}
\Gamma_{1}=\left(A_{1}+C_{1} z\right) e^{\beta z}-\left(B_{1}+D_{1} z\right) e^{-\beta z} \\
\Gamma_{2}=\left(A_{2}+C_{2} z\right) e^{\beta z}
\end{gathered}
$$


By substituting the above Galerkin vectors and their associated derivatives into the equations for stress and displacement in both layers (A5), the Boussinesq solutions become

$$
\begin{aligned}
U_{B 1}= & -i 2 \pi k_{x} \alpha_{1}\left[A_{1} \beta e^{\beta z}+B_{1} \beta e^{-\beta z}\right. \\
& \left.+C_{1}(1+\beta z) e^{\beta z}-D_{1}(1-\beta z) e^{-\beta z}\right] \\
V_{B 1}= & -i 2 \pi k_{y} \alpha_{1}\left[A_{1} \beta e^{\beta z}+B_{1} \beta e^{-\beta z}\right. \\
& \left.+C_{1}(1+\beta z) e^{\beta z}-D_{1}(1-\beta z) e^{-\beta z}\right] \\
W_{B 1}= & -\beta \alpha_{1}\left[A_{1} \beta e^{\beta z}-B_{1} \beta e^{-\beta z}+C_{1}\left(2+\beta z-2 / \alpha_{1}\right) e^{\beta z}\right. \\
& \left.+D_{1}\left(2-\beta z-2 / \alpha_{1}\right) e^{-\beta z}\right] \\
U_{B 2}= & -i 2 \pi k_{x} \alpha_{2}\left[A_{2} \beta e^{\beta z}+C_{2}(1+\beta z) e^{\beta z}\right] \\
V_{B 2}= & -i 2 \pi k_{y} \alpha_{2}\left[A_{2} \beta e^{\beta z}+C_{2}(1+\beta z) e^{\beta z}\right] \\
W_{B 2}= & -\beta \alpha_{2}\left[A_{2} \beta e^{\beta z}+C_{2}\left(2+\beta z-2 / \alpha_{2}\right) e^{\beta z}\right] \\
\tau_{x z 1}= & -i 4 \pi k_{x} \mu_{1} \alpha_{1} \beta\left[A_{1} \beta e^{\beta z}-B_{1} \beta e^{-\beta z}+C_{1}\left(2+\beta z-1 / \alpha_{1}\right) e^{\beta z}\right. \\
& \left.+D_{1}\left(2-\beta z-1 / \alpha_{1}\right) e^{-\beta z}\right] \\
\tau_{y z 1}= & -i 4 \pi k_{y} \mu_{1} \alpha_{1} \beta\left[A_{1} \beta e^{\beta z}-B_{1} \beta e^{-\beta z}+C_{1}\left(2+\beta z-1 / \alpha_{1}\right) e^{\beta z}\right. \\
& \left.+D_{1}\left(2-\beta z-1 / \alpha_{1}\right) e^{-\beta z}\right] \\
\tau_{z z 1}= & -2 \mu_{1} \alpha_{1} \beta^{2}\left[A_{1} \beta e^{\beta z}+B_{1} \beta e^{-\beta z}+C_{1}\left(3+\beta z-1 / \xi_{1}\right) e^{\beta z}\right. \\
& \left.-D_{1}\left(3-\beta z-1 / \xi_{1}\right) e^{-\beta z}\right] \\
\tau_{x z 2}= & -i 4 \pi k_{x} \mu_{2} \alpha_{2} \beta\left[A_{2} \beta e^{\beta z}+C_{2}\left(2+\beta z-1 / \alpha_{2}\right) e^{\beta z}\right] \\
\tau_{y z 2}= & -i 4 \pi k_{y} \mu_{2} \alpha_{2} \beta\left[A_{2} \beta e^{\beta z}+C_{2}\left(2+\beta z-1 / \alpha_{2}\right) e^{\beta z}\right] \\
\tau_{z z 2}= & -2 \mu_{2} \alpha_{2} \beta^{2}\left[A_{2} \beta e^{\beta z}+C_{2}\left(3+\beta z-1 / \xi_{2}\right) e^{\beta z}\right], \\
& -\beta z
\end{aligned}
$$

where $\alpha_{1}=\alpha\left(\mu_{1}, \lambda_{1}\right), \xi_{1}=\xi\left(\mu_{1}, \lambda_{1}\right), \alpha_{2}=\alpha\left(\mu_{2}, \lambda_{2}\right)$, and $\xi_{2}=\xi\left(\mu_{2}, \lambda_{2}\right)$. We can use these solutions and the appropriate boundary conditions (A1)-(A3) to solve for the six remaining Boussinesq coefficients $A_{1}, B_{1}, C_{1}, D_{1}$, $A_{2}$, and $C_{2}$. Noting the symmetry between $U_{B}, V_{B}$ and $\tau_{x z}$, $\tau_{y z}$ in equation (A10), we reduce the set of boundary conditions from nine to six:

$$
\begin{array}{ll}
U_{1}=\left.U_{2}\right|_{z=-h}, & \tau_{z z 1}=-\tau_{33}+\left.\rho g W\right|_{z=0}, \\
W_{1}=\left.W_{2}\right|_{z=-h}, & \tau_{x z 1}=\left.0\right|_{z=0}, \\
\tau_{x z 1}=\left.\tau_{x z 2}\right|_{z=-h}, \\
\tau_{z z 1}=\left.\tau_{z z 2}\right|_{z=-h} .
\end{array}
$$

This step results in six equations and six unknown Boussinesq coefficients. By substituting equations (A10) into (A11), we have the following linear system of equations, where $\psi=\alpha_{1} \beta / \tau_{33}$ :
We next invert equation (A12) to solve for the $A_{1}, B_{1}, C_{1}$, $D_{1}, A_{2}$, and $C_{2}$. The solutions for the six Boussinesq coefficients, of arbitrary elastic constants and including the gravitational restoring force, $\rho g$, are

$A_{1}=\frac{1}{d} \beta^{2} \frac{\left(\lambda_{2}+\mu_{2}\right)}{\left(\lambda_{1}+2 \mu_{1}\right)^{2}\left(\lambda_{2}+2 \mu_{2}\right)^{2}}$

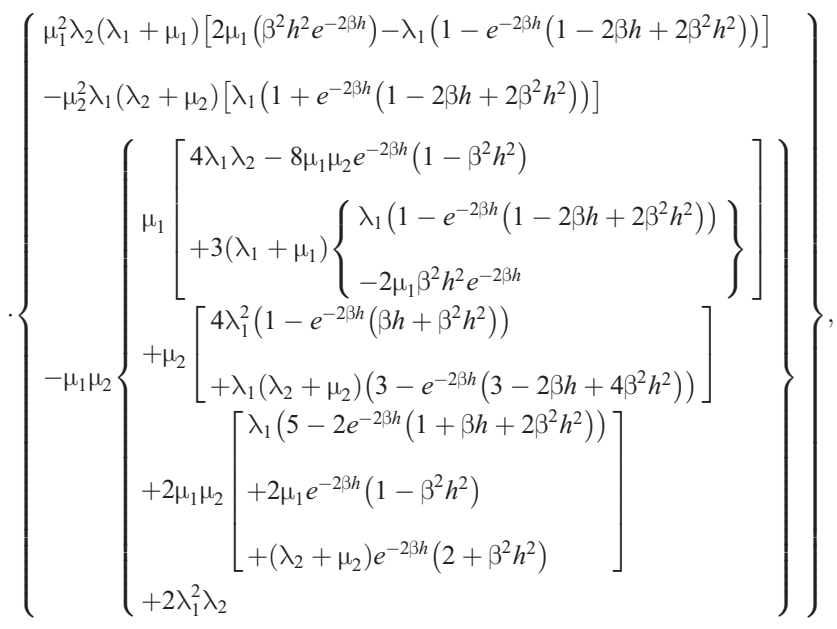

$$
\begin{aligned}
& B_{1}=\frac{1}{d} e^{-2 \beta h} \beta^{2} \frac{\left(\lambda_{2}+\mu_{2}\right)}{\left(\lambda_{1}+2 \mu_{1}\right)^{2}\left(\lambda_{2}+2 \mu_{2}\right)^{2}} \\
& \left\{\begin{array}{l}
\mu_{1}^{2} \lambda_{2}\left(\lambda_{1}+\mu_{1}\right)\left[\begin{array}{l}
\lambda_{1}\left(1+2 \beta h(1+\beta h)-e^{-2 \beta h}\right) \\
+2 \mu_{1}\left(\beta^{2} h^{2}\right)
\end{array}\right] \\
-\mu_{2}^{2} \lambda_{1}\left(\lambda_{2}+\mu_{2}\right)\left[\begin{array}{l}
\left.\lambda_{1}\left(1+2 \beta h(1+2 \beta h)+e^{-2 \beta h}\right)\right] \\
\mu_{1}\left[\begin{array}{l}
4 \lambda_{1} \lambda_{2} e^{-2 \beta h}+3 \mu_{1} \lambda_{1}\left(1+2 \beta h(1+2 \beta h)-e^{-2 \beta h}\right) \\
+3 \lambda_{1}^{2}\left(1+2 \beta h(1+\beta h)-e^{-2 \beta h}\right)
\end{array}\right] \\
-\mu_{1} \mu_{2}\left[\begin{array}{l}
4 \lambda_{1}^{2}\left(\beta h(1+\beta h)-e^{-2 \beta h}\right) \\
+\lambda_{1}\left(\lambda_{2}+\mu_{2}\right)\left(3+2 \beta h(1+2 \beta h)+3 e^{-2 \beta h}\right)
\end{array}\right] \\
{\left[\begin{array}{l}
\lambda_{1}\left(2-2 \beta h(1+2 \beta h)+3 e^{-2 \beta h}\right) \\
+2 \mu_{1}\left(1-\beta^{2} h^{2}\right) \\
-\left(\lambda_{2}+\mu_{2}\right)\left(2+\beta^{2} h^{2}\right)
\end{array}\right.} \\
+2 \mu_{1} \mu_{2}
\end{array}\right. \\
+2 \lambda_{1}^{2} \lambda_{2} e^{-2 \beta h}+6 \mu_{1}^{3} \beta^{2} h^{2}
\end{array}\right\},
\end{aligned}
$$

$$
\left[\begin{array}{l}
1 \\
0 \\
0 \\
0 \\
0 \\
0
\end{array}\right]=\left[\begin{array}{cccccc}
\psi \beta\left(2 \mu_{1} \beta-\rho g\right) & \psi \beta\left(2 \mu_{1} \beta+\rho g\right) & 2 \psi\left(\mu_{1} \beta\left(3-1 / \xi_{1}\right)-\rho g\left(1-1 / \alpha_{1}\right)\right) & -2 \psi\left(\mu_{1} \beta\left(3-1 / \xi_{1}\right)+\rho g\left(1-1 / \alpha_{1}\right)\right) & 0 & 0 \\
\beta & -\beta & \left(2-1 / \alpha_{1}\right) & \left(2-1 / \alpha_{1}\right) & 0 \\
\mu_{1} \alpha_{1} \beta e^{-\beta h} & -\mu_{1} \alpha_{1} \beta e^{\beta h} & \mu_{1} \alpha_{1}\left(2-\beta h-1 / \alpha_{1}\right) e^{-\beta h} & \mu_{1} \alpha_{1}\left(2+\beta h-1 / \alpha_{1}\right) e^{\beta h} & -\mu_{2} \alpha_{2} \beta e^{-\beta h} & -\mu_{2} \alpha_{2}\left(2-\beta h-1 / \alpha_{2}\right) e^{-\beta h} \\
\mu_{1} \alpha_{1} \beta e^{-\beta h} & \mu_{1} \alpha_{1} \beta e^{\beta h} & \mu_{1} \alpha_{1}\left(3-\beta h-1 / \xi_{1}\right) e^{-\beta h} & -\mu_{1} \alpha_{1}\left(3+\beta h-1 / \xi_{1}\right) e^{3 h} & -\mu_{2} \alpha_{2} \beta e^{-\beta h} & -\mu_{2} \alpha_{2}\left(3-\beta h-1 / \xi_{2}\right) e^{-\beta h} \\
\alpha_{1} \beta e^{-\beta h} & \alpha_{1} \beta e^{\beta h} & \alpha_{1}(1-\beta h) e^{-\beta h} & -\alpha_{1}(1+\beta h) e^{\beta h} & -\alpha_{2} \beta e^{-\beta h} & -\alpha_{2}(1-\beta h) e^{-\beta h} \\
\alpha_{1} \beta e^{-\beta h} & -\alpha_{1} \beta e^{\beta h} & \alpha_{1}\left(2-\beta h-2 / \alpha_{1}\right) e^{-\beta h} & \alpha_{1}\left(2+\beta h-2 / \alpha_{1}\right) e^{\beta h} & -\alpha_{2} \beta e^{-\beta h} & -\alpha_{2}\left(2-\beta h-2 / \alpha_{2}\right) e^{-\beta h}
\end{array}\right]\left[\begin{array}{c}
A_{1} \\
B_{1} \\
C_{1} \\
D_{1} \\
A_{2} \\
C_{2}
\end{array}\right] .
$$




$$
\begin{aligned}
& C_{1}=\frac{1}{d} \beta^{3} \frac{\left(\lambda_{1}+\mu_{1}\right)\left(\lambda_{2}+\mu_{2}\right)}{\left(\lambda_{1}+2 \mu_{1}\right)^{2}\left(\lambda_{2}+2 \mu_{2}\right)^{2}}
\end{aligned}
$$

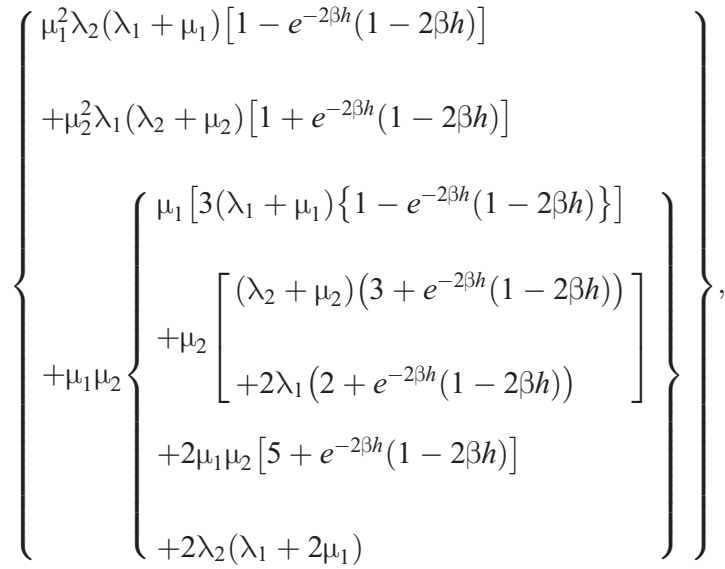

$$
D_{1}=\frac{1}{d} e^{-2 \beta h} \beta^{3} \frac{\left(\lambda_{1}+\mu_{1}\right)\left(\lambda_{2}+\mu_{2}\right)}{\left(\lambda_{1}+2 \mu_{1}\right)^{2}\left(\lambda_{2}+2 \mu_{2}\right)^{2}}
$$

$$
\left\{\begin{array}{l}
\mu_{1}^{2} \lambda_{2}\left(\lambda_{1}+\mu_{1}\right)\left[1+2 \beta h-e^{-2 \beta h}\right] \\
-\mu_{2}^{2} \lambda_{1}\left(\lambda_{2}+\mu_{2}\right)\left[1+2 \beta h+e^{-2 \beta h}\right] \\
+\mu_{1} \mu_{2}\left\{\begin{array}{l}
\mu_{1}\left[3\left(\lambda_{1}+\mu_{1}\right)\left\{1+2 \beta h-e^{-2 \beta h}\right\}\right] \\
-\mu_{2}\left[\begin{array}{l}
\left(\lambda_{2}+\mu_{2}\right)\left(1+2 \beta h-3 e^{-2 \beta h}\right) \\
+2 \lambda_{1}\left(1+2 \beta h-2 e^{-2 \beta h}\right) \\
-2 \mu_{1} \mu_{2}\left[1+2 \beta h-3 e^{-2 \beta h}\right. \\
+2 \lambda_{2}\left(\lambda_{1}+2 \mu_{1}\right) e^{-2 \beta h}
\end{array}\right.
\end{array}\right\}
\end{array}\right\}
$$

(A16)

$$
\begin{aligned}
& A_{2}=\frac{1}{d} 2 \beta^{2} \mu_{1} \frac{\left(\lambda_{1}+\mu_{1}\right)}{\left(\lambda_{2}+2 \mu_{2}\right)\left(\lambda_{1}+2 \mu_{1}\right)^{2}} \\
& \left\{\begin{array}{c}
\mu_{1}^{2}\left\{\begin{array}{c}
\mu_{2}\left[e^{-2 \beta h}\left(2 \beta^{2} h^{2}+1\right)-2 \beta h-1\right] \\
+\lambda_{2}\left[e^{-2 \beta h}(2 \beta h(\beta h-1)+1)-1\right]
\end{array}\right\} \\
-\mu_{2}^{2}\left\{\begin{array}{l}
\mu_{1}\left[e^{-2 \beta h}\left(2 \beta^{2} h^{2}+1\right)-2 \beta h-1\right] \\
+2 \lambda_{1}\left[e^{-2 \beta h}\left(\beta^{2} h^{2}\right)\right]
\end{array}\right\} \\
+\mu_{1} \mu_{2}\left\{\begin{array}{l}
\lambda_{1}\left[e^{-2 \beta h}\left(2 \beta^{2} h^{2}+1\right)-2 \beta h-1\right] \\
-2 \lambda_{2}\left[e^{-2 \beta h}(\beta h(\beta h-1)+1)-\beta h+1\right]
\end{array}\right\} \\
+\mu_{1} \lambda_{1} \lambda_{2}\left\{e^{-2 \beta h}[2 \beta h(\beta h-1)+1]-1\right\} \\
-\mu_{2} \lambda_{1} \lambda_{2}\left\{e^{-2 \beta h}[2 \beta h(\beta h-1)+1]+1\right\}
\end{array}\right\},
\end{aligned}
$$

$$
\begin{aligned}
C_{2}= & \frac{1}{d} 2 \mu_{1} \beta^{3} \frac{\left(\lambda_{1}+\mu_{1}\right)\left(\lambda_{2}+\mu_{2}\right)}{\left(\lambda_{2}+2 \mu_{2}\right)\left(\lambda_{1}+2 \mu_{1}\right)^{2}} \\
& \cdot\left\{\begin{array}{l}
\mu_{1}^{2}\left\{1-e^{-2 \beta h}(1-2 \beta h)\right\} \\
+\mu_{1} \mu_{2}\left\{3+e^{-2 \beta h}(1-2 \beta h)\right\} \\
+\mu_{1} \lambda_{1}\left\{1-e^{-2 \beta h}(1-2 \beta h)\right\} \\
+\mu_{2} \lambda_{1}\left\{1+e^{-2 \beta h}(1-2 \beta h)\right\}
\end{array}\right\},
\end{aligned}
$$

where

$$
d=\beta^{4} \frac{\left(\lambda_{1}+\mu_{1}\right)\left(\lambda_{2}+\mu_{2}\right)}{\left(\lambda_{1}+2 \mu_{1}\right)^{2}\left(\lambda_{2}+2 \mu_{2}\right)^{2}} \frac{1}{\tau_{33}}\left\{\frac{-2 \mu_{1} \beta}{\left(\lambda_{1}+2 \mu_{1}\right)} d_{1}+\rho g d_{2}\right\}
$$

and

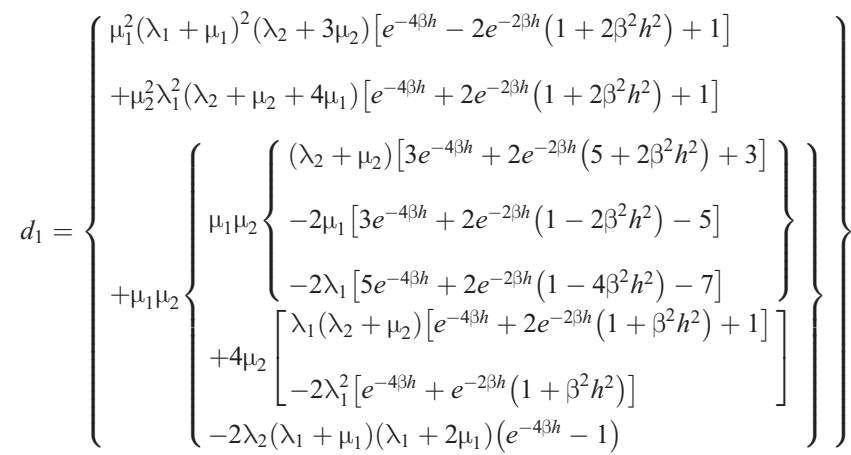

$$
d_{2}=\left\{\begin{array}{l}
-\mu_{1}^{2} \lambda_{2}\left(\lambda_{1}+\mu_{1}\right)\left[e^{-4 \beta h}-4 \beta h e^{-2 \beta h}-1\right] \\
-\mu_{2}^{2} \lambda_{1}\left(\lambda_{2}+\mu_{2}\right)\left[e^{-4 \beta h}+4 \beta h e^{-2 \beta h}-1\right] \\
+\mu_{1} \mu_{2}\left\{\begin{array}{l}
4 \lambda_{2}\left[e^{-4 \beta h}+1\right] \\
\mu_{1}\left[\begin{array}{l} 
\\
-3\left(\lambda_{1}+\mu_{1}\right)\left[e^{-4 \beta h}-4 \beta h e^{-2 \beta h}-1\right]
\end{array}\right] \\
+\mu_{2}\left[\begin{array}{l}
4 \lambda_{1}\left[e^{-4 \beta h}-2 \beta h e^{-2 \beta h}+1\right] \\
-\left(\lambda_{2}+\mu_{2}\right)\left[3 e^{-4 \beta h}+4 \beta h e^{-2 \beta h}-3\right]
\end{array}\right] \\
+2 \mu_{1} \mu_{2}\left[3 e^{-4 \beta h}-4 \beta h e^{-2 \beta h}+5\right] \\
+2 \lambda_{1} \lambda_{2}\left(e^{-4 \beta h}+1\right)
\end{array}\right\}
\end{array}\right\} .
$$

[63] These Boussinesq coefficients are greatly simplified for the case of an elastic plate overlying a fluid half-space where $\mu_{2}=0$ :

$$
\begin{gathered}
A_{1}=\frac{1}{d} \beta^{2} \frac{\left(\lambda_{1}+\mu_{1}\right)}{\left(\lambda_{1}+2 \mu_{1}\right)^{2}}\left\{2 \mu_{1}\left(\beta^{2} h^{2} e^{-2 \beta h}\right)\right. \\
\left.-\lambda_{1}\left(1-e^{-2 \beta h}\left(1-2 \beta h+2 \beta^{2} h^{2}\right)\right)\right\}, \\
B_{1}=\frac{1}{d} e^{-2 \beta h} \beta^{2} \frac{\left(\lambda_{1}+\mu_{1}\right)}{\left(\lambda_{1}+2 \mu_{1}\right)^{2}}\left\{2 \mu_{1}\left(\beta^{2} h^{2}\right)\right. \\
\left.+\lambda_{1}\left(1+2 \beta h(1+\beta h)-e^{-2 \beta h}\right)\right\}, \\
C_{1}=\frac{1}{d} \alpha_{1}^{2} \beta^{3}\left\{1-e^{-2 \beta h}(1-2 \beta h)\right\}, \\
D_{1}=\frac{1}{d} e^{-2 \beta h} \alpha_{1}^{2} \beta^{3}\left\{1+2 \beta h-e^{-2 \beta h}\right\}, \\
A_{2}=\frac{1}{d} 2 \beta^{2} \mu_{1}^{2} \alpha_{1}^{2}\left\{e^{-2 \beta h}(2 \beta h(\beta h-1)+1)-1\right\}, \\
C_{2}=\frac{1}{d} 2 \beta^{3} \mu_{1}^{2} \alpha_{1}^{2}\left\{1-e^{-2 \beta h}(1-2 \beta h)\right\},
\end{gathered}
$$

where

$$
d=-\alpha_{1}^{2} \beta^{4} \frac{1}{\tau_{33}}\left\{2 \mu_{1} \alpha_{1} \beta d_{1}+\rho g d_{2}\right\}
$$




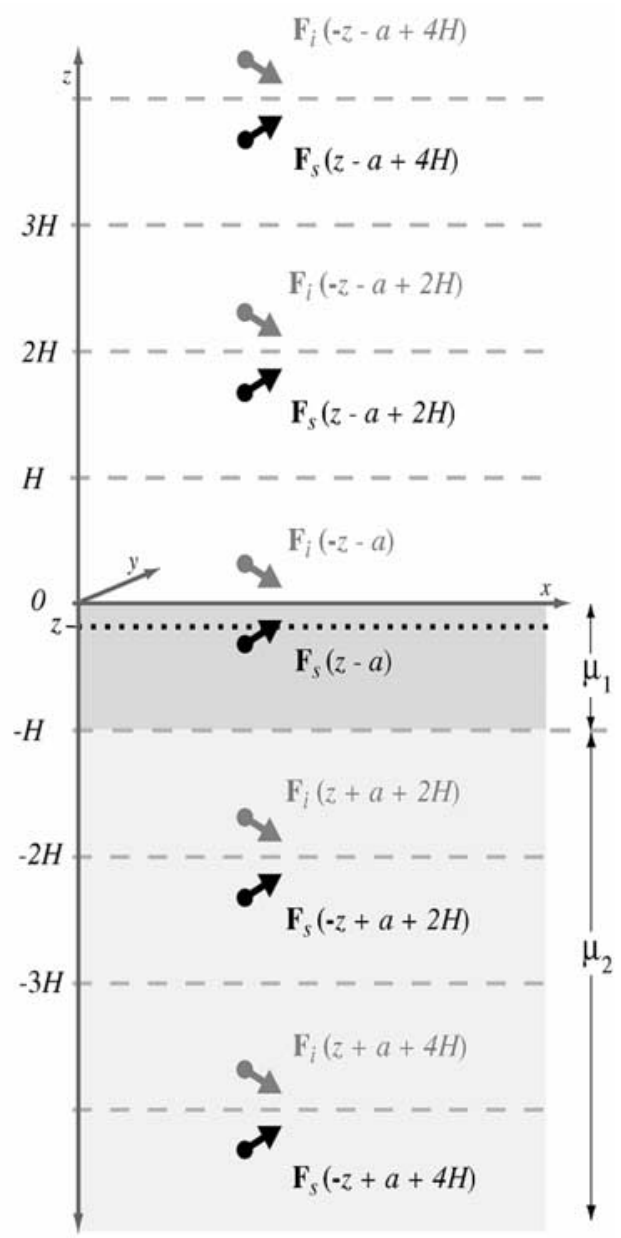

Figure B1. Sketch of the source image method for a layered elastic medium of rigidities $\mu_{1}$ (layer of thickness $H$ ) and $\mu_{2}$ (underlying half-space) used to cancel all shear stress at the surface and preserve continuity across the layer/ half-space interface. Following the method of images [Weertman, 1964], an infinite number of image terms are reflected above and below the source vector. For a halfspace model of zero rigidity (a fluid), this sum (equation (5) or (B2)) is mathematically achieved by an infinite series. These additional image contributions are represented by body forces $F_{s}$ and $F_{i}$, and are multiplied by the source and image matrices of equation (7). Note that the mathematical expressions for the image sources for $z<0$ have an opposite sign in equation (5) or (B2).

and

$$
\begin{gathered}
d_{1}=e^{-4 \beta h}-2 e^{-2 \beta h}\left(1+2 \beta^{2} h^{2}\right)+1 \\
d_{2}=e^{-4 \beta h}-4 \beta h e^{-2 \beta h}-1 .
\end{gathered}
$$

[64] In addition to the general coefficients (equations (A13)-(A21)) and those for the special case of an elastic plate overlying a fluid half-space (equations (A22)-(A30)), both including the gravitational restoring force, we have also solved for the Boussinesq coefficients for two other cases: (1) a homogeneous elastic half-space without gravity $\left(\mu_{1}=\mu_{2}, \rho g=0\right)$ and (2) a layered medium with arbitrary $\mu_{1}$ and $\mu_{2}$, also without gravity. These solutions can be found at http://topex.ucsd.edu/body_force. They have been individually validated using computer algebra and have been numerically compared to known analytic models (Figures 5 and 6).

\section{Appendix B: Method of Images}

[65] As addressed in section 2.2, for a simple homogeneous half-space model, we can use the method of images [Weertman, 1964] to place an image vector source opposite the original vector source to satisfy the requirements of a stress-free surface (equations (2) and (4)):

$$
\begin{aligned}
{\left[\begin{array}{c}
U(\mathbf{k}) \\
V(\mathbf{k}) \\
W(\mathbf{k})
\end{array}\right] } & =\mathbf{U}(\mathbf{k}, z) \\
& =\mathbf{U}_{s}(\mathbf{k}, z-a)\left[\begin{array}{c}
F_{x} \\
F_{y} \\
F_{z}
\end{array}\right]+\mathbf{U}_{i}(\mathbf{k},-z-a)\left[\begin{array}{l}
F_{x} \\
F_{y} \\
F_{z}
\end{array}\right] .
\end{aligned}
$$

In equation (B1), the $z-a$ term refers the source body force vector and the $-z-a$ term refers to the image body force vector. For a layered half-space though, we must also require that the displacements and consequent stresses remain continuous at the boundary layer between varying shear moduli. To do this, we must superpose multiple image sources, reflected both above and below the horizontal axis, to account for both the source and also the layer thickness (Figure B1). In this approach, an infinite number of secondary images, $m$, are reflected above and below the source vector, located at distances of $2 \mathrm{mH}$ (equation (5)):

$$
\begin{gathered}
{\left[\begin{array}{c}
U(\mathbf{k}) \\
V(\mathbf{k}) \\
W(\mathbf{k})
\end{array}\right]=\mathbf{U}_{s}(\mathbf{k}, z-a)\left[\begin{array}{c}
F_{x} \\
F_{y} \\
F_{z}
\end{array}\right]+\mathbf{U}_{i}(\mathbf{k},-z-a)\left[\begin{array}{c}
F_{x} \\
F_{y} \\
F_{z}
\end{array}\right]} \\
+\sum_{m=1}^{\infty}\left(\frac{\mu_{1}-\mu_{2}}{\mu_{1}+\mu_{2}}\right)^{m}\left[\begin{array}{c}
U_{i}(z-a-2 m H) \\
+U_{i}(-z-a+2 m H) \\
+U_{i}(z-a+2 m H) \\
+U_{i}(-z-a-2 m H)
\end{array}\right]\left[\begin{array}{l}
F_{x} \\
F_{y} \\
F_{z}
\end{array}\right] .
\end{gathered}
$$

In equation (B2), the $z-a \pm 2 m H$ and $-z-a \pm 2 m H$ terms are mirror images of the primary source and image, respectively. The contrasting shear moduli ratio coefficient to the left of these terms indicates the level of convergence of the layered solution. For a layer and half-space of similar elastic constants, the shear moduli coefficient 
converges easily. Yet a special case exists when the underlying half-space has a shear modulus that approaches zero (Appendix C).

\section{Appendix C: Analytic Treatment of an Infinite Sum}

[66] Inspection of equation (5) (or (B2)) shows that when the shear modulus of the half-space, $\mu_{2}$, goes to zero, the convergence of the layered solution becomes problematic. We can treat the infinite sum analytically by summing the terms from equation (8) that are dependent upon $Z: e^{-\beta Z}$ and $\beta Z e^{-\beta Z}$

[67] We first analytically treat the simple exponential, $e^{-\beta Z}$ :

$$
\sum_{m=1}^{\infty} e^{-\beta\left(z^{\prime}+2 m H\right)}=e^{-\beta z^{\prime}} e^{-\beta 2 H} \sum_{m=0}^{\infty} e^{-\beta 2 m H}=e^{-\beta z^{\prime}} \frac{e^{-\beta 2 H}}{1-e^{-\beta 2 H}},
$$

where $z^{\prime}$ now represents all terms of the form $\left( \pm z \pm d_{n}\right)$, as in those of equation (6). Next we treat the $\beta Z e^{-\beta Z}$ term by noting that

$$
-\beta \frac{\partial}{\partial \beta} e^{-\beta Z}=\beta Z e^{-\beta Z}
$$

Therefore to evaluate the sum of $\beta Z e^{-\beta Z}$, we take the derivative of the sum of $e^{-\beta Z}$ with respect to $\beta$ :

$$
\begin{aligned}
\sum_{m=1}^{\infty} \beta\left(z^{\prime}+2 m H\right) e^{-\beta\left(z^{\prime}+2 m H\right)}= & -\beta \frac{\partial}{\partial \beta} \sum_{m=1}^{\infty} e^{-\beta\left(z^{\prime}+2 m H\right)} \\
= & -\beta \frac{\partial}{\partial \beta} \frac{e^{-\beta\left(z^{\prime}+2 H\right)}}{1-e^{-\beta 2 H}} \\
= & \frac{e^{-\beta\left(z^{\prime}+2 H\right)}}{\left(1-e^{-\beta 2 H}\right)} \\
& \cdot\left[\beta\left(z^{\prime}+2 H\right)+\frac{2 \beta H e^{-\beta 2 H}}{\left(1-e^{-\beta 2 H}\right)}\right] .
\end{aligned}
$$

For the special case of an elastic plate over a fluid half-space $\left(\mu_{2}=0\right)$, computing the infinite sum of equation (5) (or (B2)) is not necessary, as equations $(\mathrm{C} 2)$ and $(\mathrm{C} 3)$ can be used instead to increase the computational convergence speed.

\section{Appendix D: Including Time Dependence: The Maxwell Model}

[68] Following the method of Nur and Mavko [1974] and Savage and Prescott [1978], we now describe our approach for the development of a time-dependent Maxwell model for 3-D displacement and stress caused by a dislocation in an elastic layer overlying a linear viscoelastic half-space. Here we develop viscoelastic coefficients that are used in conjunction with equations $(6)-(8)$ to manipulate the time dependence of the viscoelastic problem. This model will ultimately be used to demonstrate the viscoelastic response of the Earth throughout the earthquake cycle.

[69] Our theory begins with the description of the viscoelastic behavior of a Maxwell body made up of an elastic element and a viscous element, connected in series [Cohen, 1999]. The elastic and viscous element can be represented mathematically [Jaeger, 1956] by stress, $\sigma$, and stress rate, $\sigma^{\prime}$, respectively:

$$
\begin{gathered}
\sigma=\mu \varepsilon \\
\sigma^{\prime}=\eta \varepsilon^{\prime} .
\end{gathered}
$$

The elastic element (D1) describes a relationship between strain, $\varepsilon$, and the shear modulus, $\mu$, while the viscous element (D2) describes a relationship between strain rate, $\varepsilon^{\prime}$, and viscosity, $\eta$. Combining both of these linear elements for a Maxwell body in series, the constituative equation becomes

$$
\varepsilon^{\prime}=\frac{1}{\mu} \sigma^{\prime}+\frac{1}{\eta} \sigma
$$

In addition, the Maxwell time, $\tau_{m}$, is a parameter used in describing the behavior of viscoelastic relaxation. In our model, we define Maxwell time as $\tau_{m}=2 \eta / \mu$, although it should be noted that Maxwell time is defined differently by various authors (e.g., $\eta / \mu, \eta / 2 \mu$ ).

[70] We now use the Correspondence Principle for relating the constituative equation for a Maxwell body (D3) to our elastic solutions (equations (6)-(8)). According to the Correspondence Principle, when time-dependent equations are Laplace transformed, stress and strain relations retain the same "form" for all linear rheologies. Thus when the elastic solution is known, a corresponding solution describing a different rheology can be derived. Hence our first step is to take the Laplace transform of equation (D3):

$$
L\{\varepsilon\}=s \varepsilon(s)=\frac{s}{\mu} \sigma(s)+\frac{1}{\eta} \sigma(s),
$$

where $s$ is the Laplace transform variable. Solving for stress, $\sigma$, we obtain

$$
\sigma(s)=\frac{\mu s}{s+\frac{\mu}{\eta}} \varepsilon(s) .
$$

From equation (D5), we note that as Laplace variable $s$ approaches zero (zero frequency), the stress, $\sigma(s)$, goes to zero. Alternatively, as $s$ approaches infinite values, the stress takes on the form $\sigma=\mu \varepsilon$.

[71] As it is our intent to define the half-space shear modulus, $\mu_{2}$, in terms of time, we will assume $\mu=\mu_{1}$ and set

$$
\mu_{2}(s)=\frac{\mu s}{s+\frac{\mu}{\eta}}
$$

The Laplace transformed viscoelastic equation,

$$
\sigma(s)=\mu_{2}(s) \varepsilon(s)
$$


now takes on the same form as that of the purely elastic constituative equation (D1).

[72] Through the Correspondence Principle, the viscoelastic solution can be obtained as follows: (1) replace the shear modulus in the elastic solution by the Laplace transformed shear modulus variable; (2) compute the inverse transform of the layered solution; (3) integrate the solution (impulse response function) to obtain the response to a step function used to represent an earthquake; (4) identify a recursion formula for rapid and convenient calculation; (5) solve for the implied $\mu_{2}$ associated with each image in the infinite layers; (6) ensure the bulk modulus remains constant by varying $\lambda_{2}$ for each $\mu_{2}$.

[73] Our goal is to map time $(t)$ and viscosity $(\eta)$ into an implied $\mu_{2}$ and then solve for the corresponding elastic constant $\lambda_{2}$ by requiring a constant bulk modulus. We have already shown that the layered elastic solution may be described by adding the sources and images associated with the layer in an infinite series (equations (5)-(6)). We now focus on the treatment of the shear modulus ratio inside the infinite series solution, which we will now refer to as $\chi$ :

$$
\chi=\frac{\mu_{1}-\mu_{2}}{\mu_{1}+\mu_{2}} .
$$

We substitute equation (D6) into (D8) and again assume $\mu=$ $\mu_{1}$. The Laplace transform of $\chi$ then becomes

$$
\chi(s)=\frac{\mu-\frac{\mu s}{s+\mu / \eta}}{\mu+\frac{\mu s}{s+\mu / \eta}}=\frac{\frac{\mu}{2 \eta}}{s+\frac{\mu}{2 \eta}} .
$$

By setting $a=\mu / 2 \eta=1 / \tau_{m}$, the inverse of the Maxell time, we find

$$
\chi(s)=\frac{a}{s+a} .
$$

Because the infinite sum of equations (5)-(6) is raised to the power of $m$, we next take the inverse Laplace Transform of $\chi^{m}(s)$,

$$
\chi^{m}(t)=\frac{a^{m} t^{m-1}}{(m-1) !} e^{-a t}
$$

which is the impulse response function. We next integrate this impulse response function to obtain the response to a step function, $H(t)$, which represents seismic faulting events over time. Let coefficients $A_{m}(t)$ and $B_{m}(t)$ describe this behavior:

$$
\int_{0}^{t} \chi^{m}(t) d t=\frac{a^{m}}{(m-1) !} \int_{0}^{t} t^{m-1} e^{-a t} d t=A_{m}(t)
$$

and

$$
B_{m}(t)=\frac{(m-1) !}{a^{m}} A_{m}(t),
$$

where

$$
B_{m}(t)=\int_{0}^{t} t^{m-1} e^{-a t} d t
$$

Integrating by parts reveals the following recursion formula:

$$
B_{m}=-\frac{t^{m-1}}{a} e^{-a t}+\frac{m-1}{a} B_{m-1},
$$

where

$$
B_{1}=\int_{0}^{t} e^{-a t} d t=\frac{1}{a}\left[1-e^{-a t}\right] .
$$

Substituting $a=\mu / 2 \eta=1 / \tau_{m}$ into D12 and D14, the first three terms in this infinite series are

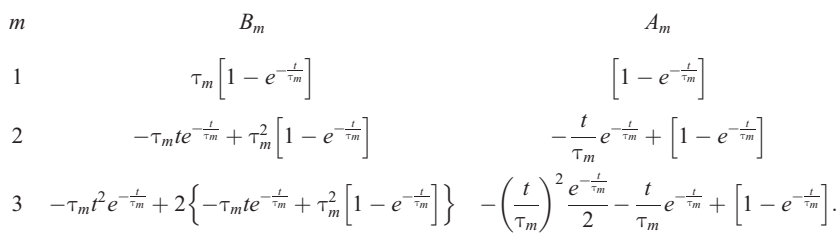

[74] We next replace the time-integrated shear modulus ratio (D8) with the new $A_{m}$ coefficients, where each image source will have its own implied $\mu_{2}$ :

$$
\left(\frac{\mu_{1}-\mu_{2}}{\mu_{1}+\mu_{2}}\right)^{m}=A_{m}
$$

Solving for the implied $\mu_{2}$ corresponding to each $A_{m}$ coefficient, we obtain

$$
\mu_{2}=\mu_{1}\left(\frac{1-A_{m}^{\frac{1}{m}}}{1+A_{m}^{\frac{1}{m}}}\right)
$$

In addition to the time-dependent behavior of the effective shear modulus of the viscoelastic half-space, we also must ensure that the bulk modulus, $\kappa=\lambda+\frac{2}{3} \mu$, remains constant:

$$
\kappa_{2}=\lambda_{2}+\frac{2}{3} \mu_{2}=\text { constant }
$$

If we set

$$
\mu_{2}=\mu_{1}\left(\frac{1-A_{m}^{\frac{1}{m}}}{1+A_{m}^{\frac{1}{m}}}\right)
$$

then

$$
\lambda_{2}=\kappa_{2}-\frac{2}{3} \mu_{1}\left(\frac{1-A_{m}^{\frac{1}{m}}}{1+A_{m}^{\frac{1}{m}}}\right) .
$$


[75] In addition, we must also note that the vertical Boussinesq load assumes the shear response of a single Maxwell time. Since we have only solved the vertical loading problem for an elastic plate overlying a viscoelastic half-space, we must select the most appropriate viscosity or Maxwell time. We choose the viscosity of the uppermost image $(m=1)$, noting that this is an approximation to the exact layered behavior. This approach follows the timedependent loading problem discussed by Brotchie and Silvester [1969] where loading is scaled by a similar viscosity-dependent coefficient. If a vertical load is applied at $t=0$ and the initial elastic response is described as $W(\mathbf{k}, 0)$, then the long-term response of the elastic plate over a fluid half-space is $W(\mathbf{k}, \infty)$. For a Maxwell time of $\tau_{m}=$ $2 \eta / \mu$, the viscoelastic response becomes

$$
\begin{aligned}
W(\mathbf{k}, t) & =W(\mathbf{k}, 0)+\left[1-e^{-\frac{t}{\tau_{m}}}\right][W(\mathbf{k}, \infty)-W(\mathbf{k}, 0)] \\
& =W(\mathbf{k}, 0) e^{-\frac{t}{\tau_{m}}}+\left[1-e^{-\frac{t}{\tau_{m}}}\right] W(\mathbf{k}, \infty)
\end{aligned}
$$

By assuming $\mu_{2}(t)$ from $A_{m}(1)$, the Maxwell coefficients become

$$
A_{m}(1)=\left(1-e^{-\frac{t}{\tau_{m}}}\right)
$$

and

$$
\mu_{2}=\mu_{1}\left(\frac{e^{-\frac{t}{\tau_{m}}}}{2-e^{-\frac{t}{\tau_{m}}}}\right)
$$

As a check, we can verify that shear time variations are consistent with those expected for end-member models. For example, for times approaching zero, shear moduli of the layer and half-space are equal $\left(\mu_{1}=\mu_{2}\right)$. Alternatively, for times approaching infinity, the shear modulus of the half-space goes to zero $\left(\mu_{2}=0\right)$. The 2-D models of Figures $3 a-3 b$ demonstrate this behavior.

\section{Appendix E: Force Couples on a Regular Grid}

[76] A dislocation in a fault plane is commonly represented by body force couples. In the case of a horizontal strike-slip fault, a double-couple should be used to ensure local balanced moment in the horizontal plane [Burridge and Knopoff, 1964]. Here we describe the algorithm for generating single- and double-couple body forces for a segmented fault trace mapped onto a regular grid (Figure 1).

[77] Consider a grid with cell spacing $\Delta x$. The final displacement and stress model cannot resolve features smaller than the cell spacing, thus we approximate a fault segment with a finite length $L$, oriented along the $x$ axis, and a finite thickness $\sigma \geq \Delta x$ as follows:

$$
f(x, y)=g(x) h(y)
$$

where the across-fault function $h(y)$ is a Gaussian function:

$$
h(y)=\frac{1}{\sigma \sqrt{2 \pi}} \exp \left(\frac{y^{2}}{2 \sigma^{2}}\right)
$$

We represent a curved fault trace as a large number of straight overlapping segments of the form

$$
g(x)=\left\{\begin{array}{cc}
\frac{1}{2}\left[1-\cos \frac{\pi(x+2 \Delta x)}{4 \Delta x}\right] & -2 \Delta x<x<2 \Delta x \\
1 & 2 \Delta x<x<L-2 \Delta x \\
\frac{1}{2}\left[1-\cos \frac{\pi(L+2 \Delta x-x)}{4 \Delta x}\right] & L-2 \Delta x<x<L+2 \Delta x
\end{array}\right.
$$

where $x$ is the distance from the start of the segment and $L$ is the segment length. The segments are arranged end-to-end so that the sum of the overlapping cosine functions equals one. The spatial variations in the force-couple are constructed by taking the derivatives of the fault function. The primary couple is parallel to the fault ( $x$ direction) and corresponds to the fault-normal derivative

$$
f_{1}(x, y)=g(x) \frac{\partial h}{\partial y}
$$

where

$$
\frac{\partial h}{\partial y}=\frac{-y}{\sigma^{3} \sqrt{2 \pi}} \exp \left(\frac{y^{2}}{2 \sigma^{2}}\right)
$$

The secondary force couple is perpendicular to the fault ( $y$ direction) and corresponds to the fault-parallel derivative

$$
f_{2}(x, y)=\frac{\partial g}{\partial x} h(y)
$$

where

$$
\frac{\partial g}{\partial x}=\left\{\begin{array}{cc}
\frac{\pi}{8 \Delta x} \sin \frac{\pi(x+2 \Delta x)}{4 \Delta x} & -2 \Delta x<x<2 \Delta x \\
1 & 2 \Delta x<x<L-2 \Delta x \\
\frac{-\pi}{8 \Delta x} \sin \frac{\pi(L+2 \Delta x-x)}{4 \Delta x} & L-2 \Delta x<x<L+2 \Delta x
\end{array} .\right.
$$

A rotation matrix is used to rotate the force couple functions to the proper orientation:

$$
\left(\begin{array}{l}
x \\
y
\end{array}\right)=\left(\begin{array}{cc}
\cos \theta & -\sin \theta \\
\sin \theta & \cos \theta
\end{array}\right)\left(\begin{array}{l}
x^{\prime} \\
y^{\prime}
\end{array}\right),
$$

where $\theta$ is the angle between the $x$ axis and the fault trace $(\theta>0$ represents counterclockwise rotation).

[78] Three modes of displacement can be applied on each fault segment: $F_{1}$ is strike slip, $F_{2}$ is dip slip, and $F_{3}$ is opening of the fault. Once the primary and secondary force couple functions are computed and rotated into the fault direction, they are multiplied by the strength of the dislocation to form three grids corresponding to the $F_{1}, F_{2}$, and $F_{3}$ modes. These three force components must then be 
rotated into the Cartesian frame $F_{x}, F_{y}$, and $F_{z}$ using the following formulas:

$$
\left(\begin{array}{c}
F_{x}(x, y) \\
F_{y}(x, y) \\
F_{z}(x, y)
\end{array}\right)=f_{1}(x, y)\left(\begin{array}{ccc}
-\cos \theta & 0 & \sin \theta \\
\sin \theta & 0 & \cos \theta \\
0 & 1 & 0
\end{array}\right)\left(\begin{array}{l}
F_{1} \\
F_{2} \\
F_{3}
\end{array}\right) .
$$

Balancing of the moment due to the horizontal strike-slip force couple $F_{1}$ requires a second force component given by

$$
\left(\begin{array}{l}
F_{x}^{2}(x, y) \\
F_{y}^{2}(x, y)
\end{array}\right)=f_{2}(x, y)\left(\begin{array}{c}
F_{1} \sin \theta \\
F_{1} \cos \theta
\end{array}\right) .
$$

Note that this force couple only applies to the end of each fault segment and the forces largely cancel where fault segments abut as described in Burridge and Knopoff [1964]. The moment generated by the vertical dip-slip $F_{2}$ and the opening $F_{3}$ of the fault will produce topography that will balance the moment under the restoring force of gravity.

[79] Acknowledgments. We thank Steve Cohen, Associate Editor Joan Gomberg, and an anonymous reviewer for their help on improving the manuscript and clarifying the model. We also thank Yuri Fialko and Debi Kilb for providing careful in-house reviews of draft versions of the manuscript. This research was supported by the NASA Solid Earth and Natural Hazards Program (NAGS-9623), the NASA Earth System Science Fellowship Program (ESSF-0131), and the NSF Earth Science Program (EAR-0105896).

\section{References}

Anderson, G., B. Aagaard, and K. Hudnut (2003), Fault interactions and large complex earthquakes in the Los Angeles area, Science, 302, 19461949.

Boussinesq, J. (1885), Application des Potentiels a l'Etude de l'Equilibre et du Mouvement des Solides Elastiques, 508 pp., Gauthier-Viallars, Paris. Brotchie, J. F., and R. Silvester (1969), On crustal flexure, J. Geophys. Res., $74,5240-5252$.

Burmister, D. M. (1943), The theory of stresses and displacements in layered systems and applications of the design of airport runways, paper presented at the Annual Meeting, Highway Res. Board Natl. Res. Counc., Washington, D. C.

Burridge, R., and L. Knopoff (1964), Body force equivalents for seismic dislocations, Bull. Seismol. Soc. Am., 54, 1875-1888.

Chinnery, M. A. (1961), The deformation of the ground around surface faults, Bull. Seismol. Soc. Am., 50, 355-372.

Cohen, S. C. (1984), Postseismic deformation due to subcrustal viscoelastic relaxation following dip-slip earthquakes, J. Geophys. Res., 89, 45384544.

Cohen, S. C. (1999), Numerical models of crustal deformation in seismic zones, Adv. Geophys., 41, 133-231.

Crouch, S. L., and A. M. Starfield (1983), Boundary Element Methods in Solid Mechanics, 322 pp., Allen and Unwin, Concord, Mass.

Deng, J., M. Gurnis, H. Kanamori, and E. Hauksson (1998), Viscoelastic flow in the lower crust after the 1992 Landers, California, earthquake, Science, 282, 1689-1692.

Deng, J., K. Hudnut, M. Gurnis, and E. Hauksson (1999), Stress loading from viscous flow in the lower crust and triggering of aftershocks following the 1994 Northridge, California, earthquake, Geophys. Res. Lett., 26, $3209-3212$.

Fialko, Y. (2004a), Evidence of fluid-filled upper crust from observations of postseismic deformation due to the $1992 M_{w} 7.3$ Landers earthquake, J. Geophys. Res., 109, B08401, doi:10.1029/2004JB002985.

Fialko, Y. (2004b), Probing the mechanical properties of seismically active crust with space geodesy: Study of the coseismic deformation due to the $1992 M_{w} 7.3$ Landers (southern California) earthquake, J. Geophys. Res., 109, B03307, doi:10.1029/2003JB002756.

Frankel, A. (1993), Three-dimensional simulations of the ground motions in the San Bernardino valley, California, for hypothetical earthquakes on the San Andreas fault, Bull. Seismol. Soc. Am., 83, 1020-1041.
Freed, A. M., and J. Lin (1998), Time-dependent changes in failure stress following thrust earthquakes, J. Geophys. Res., 103, 24,393-24,409.

Freed, A. M., and J. Lin (2001), Delayed triggering of the 1999 Hector Mine earthquake by viscoelastic stress transfer, Nature, 411, 180-183.

Harris, R. A. (1998), Introduction to special section: Stress triggers, stress shadows, and implications for seismic hazard, J. Geophys. Res., 103, $24,347-24,358$.

Harris, R. A., and R. W. Simpson (1993), In the shadow of 1857: An evaluation of the static stress changes generated by the M8 Ft. Tejon, California, earthquake (abstract), Eos Trans. AGU, 74(43), Fall Meet. Suppl., 427.

Harris, R. A., and R. W. Simpson (1996), In the shadow of 1857: The effect of the great Fort Tejon earthquake on subsequent earthquakes in southern California, Geophys. Res. Lett., 23, 229-232.

Harris, R. A., and R. W. Simpson (1998), Suppression of large earthquakes by stress shadows: A comparison of Coulomb and rate-and-state failure, J. Geophys. Res., 103, 24,439-24,452.

Haskell, N. A. (1953), The dispersion of surface waves on multilayered media, Bull. Seismol. Soc. Am., 54, 17-34.

Hearn, E. H., B. H. Hager, and R. E. Reilinger (2002), Viscoelastic deformation from North Anatolian Fault Zone earthquakes and the eastern Mediterranean GPS velocity field, Geophys. Res. Lett., 29(11), 1549, doi:10.1029/2002GL014889.

Heki, K., S. Miyazaki, and H. Tsuji (1997), Silent fault slip following an interplate thrust earthquake at the Japan Trench, Nature, 386, 595-598.

Ivins, E. R. (1996), Transient creep of a composite lower crust: 2. A polymineralic basis for rapidly evolving postseismic deformation modes, J. Geophys. Res., 101, 28,005-28,028.

Jaeger, J. C. (1956), Elasticity, Fracture, and Flow, 102 pp., Metheun, New York.

Kenner, S., and P. Segall (1999), Time-dependence of the stress shadowing effect and its relation to the structure of the lower crust, Geology, 27, $119-122$.

Kilb, D., J. Gomberg, and P. Bodin (2000), Triggering of earthquake aftershocks by dynamic stresses, Nature, 408, 570-574.

Kilb, D., J. Gomberg, and P. Bodin (2002), Aftershock triggering by complete Coulomb stress changes, J. Geophys. Res., 107(B4), 2060, doi:10.1029/2001JB000202.

King, G. C. P., and M. Cocco (2001), Fault interaction by elastic stress changes: New clues from earthquake sequences, Adv. Geophys., 44, 138 .

King, G. C. P., R. S. Stein, and J. Lin (1994), Static stress changes and the triggering of earthquakes, Bull. Seismol. Soc. Am., 84, 935-953.

Le Pinchon, X., J. Francheteau, and J. Bonnin (1973), Developments in Geotectonics, vol. 6, Plate Tectonics, Elsevier Sci., New York.

Love, A. E. H. (1929), The stress produced in a semi-infinite solid by pressure on part of the boundary, Philos. Trans. R. Soc. London, Ser. A, $667,377-420$.

Love, A. E. H. (1944), The Mathematical Theory of Elasticity, 2nd ed., Cambridge Univ. Press, New York.

Lysmer, J., and L. A. Drake (1972), A finite element method for seismology, in Methods in Computational Physics, edited by B. Alder, S. Fernbach, and B. A. Bolt, pp. 181-216, Academic, San Diego, Calif.

Massonnet, D., W. Thatcher, and H. Vadon (1996), Radar interferometry detects two mechanism of postseismic relaxation following the Landers, California earthquake, Nature, 382, 612-616.

McKenzie, D., and C. Bowin (1976), The relationship between bathymetry and gravity in the Atlantic Ocean, J. Geophys. Res., 81, 1903-1915.

Melosh, H. J. (1983), Vertical movements following a dip-slip earthquake, Geophys. Res. Lett., 10, 47-50.

Nur, A., and G. Mavko (1974), Postseismic viscoelastic rebound, Science, 183, 204-206.

Okada, Y. (1985), Surface deformation due to shear and tensile faults in a half-space, Bull. Seismol. Soc. Am., 75, 1135-1154.

Okada, Y. (1992), Internal deformation due to shear and tensile faults in a half-space, Bull. Seismol. Soc. Am., 82, 1018-1040.

Olsen, K. B., and G. T. Schuster (1992), Site amplification in the Salt Lake Valley by three-dimensional elastic wave propagation, Eos Trans. AGU, 73, 338 .

Parsons, T. (2002), Post-1906 stress recovery of the San Andreas fault system calculated from three-dimensional finite element analysis, J. Geophys. Res., 107(B8), 2162, doi:10.1029/2001JB001051.

Peltzer, G. P., P. Rosen, F. Rogez, and K. Hudnut (1996), Postseismic rebound in fault step-overs caused by pore fluid flow, Science, 273, $1202-1204$.

Peltzer, G., P. Rosen, F. Rogez, and K. Hudnut (1998), Poroelastic rebound along the Landers 1992 earthquake surface rupture, J. Geophys. Res., $103,30,131-30,145$.

Pollitz, F. F., G. Peltzer, and R. Bürgmann (2000), Mobility of continental mantle: Evidence from postseismic geodetic observations following the 1992 Landers earthquake, J. Geophys. Res., 105, 8035-8054. 
Pollitz, F. F., C. Wicks, and W. Thatcher (2001), Mantle flow beneath a continental strike-slip fault: Postseismic deformation after the 1999 Hector Mine earthquake, Science, 293, 1814-1818.

Rundle, J. B., and D. D. Jackson (1977), A three-dimensional viscoelastic model of a strike slip fault, Geophys. J. R. Astron. Soc., 49, 575-591.

Rybicki, K. (1971), The elastic residual field of a very long strike-slip fault in the presence of a discontinuity, Bull. Seismol. Soc. Am., 61, 72-92.

Sato, R. (1971), Crustal deformation due to a dislocation in a multi-layered medium, J. Phys. Earth, 19, 31-46.

Savage, J. C., and W. H. Prescott (1978), Asthenosphere readjustment and the earthquake cycle, J. Geophys. Res., 83, 13,369-13,376.

Savage, J. C., and J. L. Svarc (1997), Postseismic deformation associated with the $1992 \mathrm{M}_{\mathrm{w}}=7.3$ Landers earthquake, southern California, J. Geophys. Res., 102, 7565-7577

Shen, Z., D. Jackson, Y. Feng, M. Cline, M. Kim, P. Fang, and Y. Bock (1994), Postseismic deformation following the Landers earthquake, California, 28 June 1992, Bull. Seismol. Soc. Am., 84, 780-791.

Singh, S. J. (1970), Static deformation of a multilayered half-space by internal sources, J. Geophys. Res., 75, 3257-3263.

Smith, B. R., and D. T. Sandwell (2003), Coulomb stress accumulation along the San Andreas Fault system, J. Geophys. Res., 108(B6), 2296, doi:10.1029/2002JB002136.

Stein, R. S., G. C. P. King, and J. Lin (1994), Stress triggering of the 1994 $\mathrm{M}=6.7$ Northridge, California, earthquake by its predecessors, Science, $265,1432-1435$

Steketee, J. A. (1958), On Volterra's dislocations in a semi-infinite elastic medium, Can. J. Phys., 36, 192-205.

Thatcher, W. (1983), Nonlinear strain buildup and the earthquake cycle on the San Andreas Fault, J. Geophys. Res., 88, 5893-5902.

Thatcher, W. (1984), The earthquake cycle at the Nankai Trough, southwest Japan, J. Geophys. Res., 89, 3087-3101.

Thomas, A. L. (1993), Poly3D: A three-dimensional, polygon element, displacement discontinuity boundary element computer program with applications to fractures, faults, and cavities in the Earth's crust, M.S. thesis, Stanford Univ., Stanford, Calif.

Timoshenko, S. (1934), Theory of Elasticity, McGraw-Hill, New York.
Toda, S., R. Stein, and T. Sagiya (2002), Evidence from the AD 2000 Izu Islands earthquake swarm that stressing rate governs seismicity, Nature, $419,58-61$

Turcotte, D. L., and G. Schubert (1982), Geodynamics, 1st ed., Cambridge Univ. Press, New York.

Wang, R., F. Martin, and F. Roth (2003), Computation of deformation induced by earthquakes in a multi-layered elastic crust-FORTRAN programs EDGRN/EDCMP, Comp. Geosci., 29, 195-207.

Ward, S. N. (1984), A note on lithospheric bending calculations, Geophys. J. R. Astron. Soc., 78, 241-253.

Ward, S. N. (1985), Quasi-static propagator matrices: Creep on strike-slip faults, Tectonophysics, 120, 83-106.

Wdowinski, S., Y. Bock, J. Zhang, P. Fang, and J. Genrich (1997), Southern California permanent GPS Geodetic Array: Spatial filtering of daily positions for estimating coseismic and postseismic displacements induced by the 1992 Landers earthquake, J. Geophys. Res., 102, 18,057-18,070.

Weertman, J. (1964), Continuum distribution of dislocations on faults with finite friction, Bull. Seismol. Soc. Am., 54, 1035-1058.

Williams, C. A., and R. M. Richardson (1991), A rheologically layered three-dimensional model of the San Andreas fault in central and southern California, J. Geophys. Res., 96, 16,597-16,623.

Yang, M., and M. N. Toksoz (1981), Time dependent deformation and stress relaxation after strike slip earthquakes, J. Geophys. Res., 86, 2889-2901.

Zang, L., and A. K. Chopra (1991), Three-dimensional analysis of spatially varying ground motions around a uniform canyon in a homogeneous half space, Earthquake Eng. Struct. Dyn., 20, 911-926.

Zeng, Y. (2001), Viscoelastic stress-triggering of the 1999 Hector Mine earthquake by the 1992 Landers earthquake, Geophys. Res. Lett., 28, $3007-3010$

D. Sandwell and B. Smith, Institute for Geophysics and Planetary Physics, Scripps Institution of Oceanography, 9500 Gilman Drive, Mail Code 0225, La Jolla, CA 92093, USA. (dsandwell@ucsd.edu; brsmith@ucsd.edu) 